\title{
„Prächtiges Menschenmaterial“ - Anthropometrische Konstitutionsforschung auf der Suche nach dem statistischen Normalkörper (1914-1922)
}

\author{
Nadine Metzger
}

"Splendid Human Material"-Anthropometric Constitutional Research to Statistically Determine the Normal Human Body (1914-1922)

At the center of this work stands the anthropometric research program during World War I for studying constitutional medicine and the connected series of investigations by the medical internists Theodor Brugsch, Hermann Rautmann and Max Berliner, their advances in the statistics of variability as well as the subsequent debate in constitutional medicine and pathology on the definition of the physical norm.

In order to create a data basis for the "normal" body in the study of constitutional medicine, a series of young German internists undertook comprehensive anthropometric studies in the context of World War I, thereby taking advantage of the opportunity offered them by war to conduct a series of examinations of soldiers, but without having first reflected on methods of measurement, comparison, and evaluation. At the same time, the concept of the "normal" body, then only vaguely formed, still needed to be critically expounded. However, this changed during the subsequent period and led not only to a stronger emphasis on methodology, rather also to greater competency in mathematical statistics and philosophical cogitation on the meaning of the "norm". In this way, constitutional medicine originated the potent medical norm debate of the early 1920s which still resonates in medical theory today. By this means the few years following the end of World War I not only represented a turning point for constitutional medicine regarding the reflective use of methodology, but also introduced a new orientation of their research questions: away from the "normal" body to individuality.

Keywords: Constitutional medicine, Medical statistics, Medical norm, Theodor Brugsch, Hermann Rautmann, Max Berliner

Im Zentrum der vorliegenden Arbeit steht das anthropometrische Forschungsprogramm der Konstitutionslehre während des Ersten Weltkrieges und die davon angestoßenen Reihenuntersuchungen der Internisten Theodor Brugsch, Hermann Rautmann und Max Berliner, deren Vorstöße in die Variabilitätsstatistik sowie die anschlieBende konstitutionspathologische Debatte um die Definition einer körperlichen Norm.

Um der Konstitutionslehre eine Datengrundlage für den „Normkörper" zu schaffen, unternahm im Umfeld des Ersten Weltkrieges eine Reihe junger deutscher Internisten umfassende anthropometrische Studien und nutzte dabei die Gelegenheit, die innen der Krieg zu Reihenuntersuchungen an Soldaten bot, ohne vorher über Messmethoden, Vergleichs- und Auswertungsmöglichkeiten zu reflektieren. Dies änderte sich jedoch in der Folgezeit und führte nicht nur zu einem starken Zuwachs an methodischer sondern auch an mathematischstatistischer Kompetenz. Zudem problematisierte das konstitutionspathologische Projekt den Normbegriff, was in der intensiven Normdebatte der 1920er Jahre mündete. Damit stellen die wenigen Jahre nach Ende des Ersten Weltkriegs bis 1922 für die Konstitutionslehre nicht nur einen Drehpunkt hinsichtlich des reflektierten Methodengebrauchs dar, sondern leiteten auch eine Neuausrichtung inrer Forschungsfragen ein: weg vom „Normkörper" hin zur Individualität. 
Die Ansammlung metrischer Daten sollte der noch jungen Konstitutionslehre eine größtmögliche Orientierung an naturwissenschaftlichen Methoden sicherstellen: Messen, Zählen und Wiegen galten als der Königsweg, um ihr seit 1911 interdisziplinär aufgestelltes Forschungsprogramm auf eine solide wie moderne wissenschaftliche Basis zu stellen: Wie beeinflussen individuelle Faktoren des Patientenkörpers Krankheitsentstehung und -verläufe? Welche körperlichen Zeichen, sogenannte „Konstitutionsanomalien“, weisen auf bestimmte Krankheitsneigungen hin? Um diese Fragen überhaupt beantworten zu können, musste sich die Konstitutionsforschung zunächst ein klares Bild des „normalen“ gesunden Körpers verschaffen. Hierzu dienten unter anderem anthropometrische Reihenuntersuchungen, die von konstitutionspathologisch ${ }^{1}$ motivierten Internisten während des Ersten Weltkrieges sowie kurz danach durchgeführt wurden und die beispielhaft im Zentrum der vorliegenden Arbeit stehen.

Diese anthropometrischen Untersuchungen können einerseits als typisch für die konstitutionspathologische Episteme während des Ersten Weltkrieges gelten, während sie andererseits entscheidende epistemologische und medizintheoretische Entwicklungen für die gesamte Medizin anstießen, wie im Folgenden entwickelt wird. Dazu werden die Studien der drei Internisten Theodor Brugsch (1878-1963), Hermann Rautmann (1885-1956) und Max Berliner (1888-1960/65) analysiert, die während beziehungsweise im Anschluss an den Ersten Weltkrieg durchgeführt wurden (Brugsch 1917; Brugsch 1918; Berliner 1920a, b; Berliner 1921; Rautmann 1921): Welcher anthropometrischer Methoden bedienten sich diese drei Konstitutionspathologen und wie verorteten sie sich in zeitgenössischen Messtraditionen? Wie werteten sie die gewonnenen Daten aus und wie tief stiegen sie in die zeitgenössische "mathematische“ Statistik ${ }^{2}$ ein, also in mathematische Verfahren zur Analyse von Verteilungen und Korrelationen auf der Grundlage von Wahrscheinlichkeitsrechnung und Fehlertheorie (Žižek 1923: 8)? Welche Antworten formulierten sie auf die Frage nach „normaler" Konstitution? Wie schritten sie fort vom Messen zur Beschäftigung mit Statistik bis hin zur Problematisierung der Norm? Um diese Fragen auch für die Konstitutionslehre als solche beantworten zu können, werden außerdem konstitutionspathologische Lehr- und Überblickswerke von 1914 bis 1922 ausgewertet (Martius 1914; Bauer 1921; Bauer 1923; Grote 1921; Günther 1922) sowie Spezialveröffentlichungen zu den einzelnen Punkten herangezogen. 
Der überwiegende Anteil der untersuchten anthropometrischen Arbeiten entstand im Kontext des Ersten Weltkrieges. So bot sich dem Freiburger Internisten und aufstrebenden Konstitutionspathologen Hermann Rautmann vom Frühherbst 1917 bis zum Herbst 1918 die Gelegenheit, im Rahmen von Tauglichkeitsprüfungen für den Kriegsdienst fast zweitausend junge und weitestgehend gesunde Männer $\mathrm{zu}$ vermessen. Wie viele andere Nachwuchswissenschaftler auch folgte er dabei dem allgemeinen Ruf forschungsorientierter Mediziner, das „Experimentierfeld des Krieges“ für umfassende Datengewinnung zu nutzen - die Gelegenheit für konstitutionspathologische Grundlagenforschung schien einmalig. Im April 1916 hatte Rautmanns Freiburger Förderer, der Pathologe Ludwig Aschoff (1866-1942), auf der „Kriegspathologischen Tagung“ seine Kollegen eingeschworen auf ,jene große Aufgabe, [...] nämlich eine gesicherte Unterlage für die Konstitutionslehre zu schaffen" (Aschoff 1916: 3), und Rautmanns Chef Oskar de la Camp (1871-1925) forderte die „restlos[e] und fruchtbringend $[\mathrm{e}]$ “ Verwertung der Tatsachen, „die der Krieg auch für die [...] Konstitutionsforschung gezeitigt hatte" (de la Camp 1918: 517). Die großangelegte konstitutionspathologische Datensammlung arbeitete mit sehr langfristiger Perspektive: De la Camp (1918: 517) sprach für die Auswertung von ,jahre-, vielleicht jahrzehnte langer Arbeit“. Wenig Gedanken machte man sich angesichts des verheißungsvollen Datenüberflusses hingegen darüber, wie die Daten ausgewertet werden sollten und was sie überhaupt auszusagen vermochten - ein prinzipielles Problem datengenerierender medizinischer Forschung von einiger Aktualität.

Der ausgebrochene Krieg verschaffte Konstitutionsforschern, wie viele ihrer Kollegen im militärärztlichen Einsatz, einen leichten Zugang zu "prächtige[m] Menschenmaterial“ (Rössle 1919: 19) - lebenden wie toten Körpern junger, weitestgehend gesunder Männer in großer Zahl. Im Kontext von Musterungen konnten tausende von Soldaten von einem Arzt vermessen werden, so wie dies Rautmann tat, in Lazaretten konnte auf krankheitsspezifischen Stationen nach konstitutionellen Zeichen für eine Neigung zu bestimmten Infektionskrankheiten gesucht werden und die Kriegspathologie betrieb zentral koordinierte Prosekturen an allen Fronten, um möglichst viel Erkenntnisgewinn aus den Kriegstoten ziehen zu können (Prüll 1996). Doch insbesondere bei den gesammelten anthropometrischen Daten wurde schnell klar, dass es an methodischem Rüstzeug wie Kenntnissen der mathematischen Statistik fehlte, um diese gewinnbringend verarbeiten zu können. Aus der Datenerhebung entstanden, verstand sich Rautmanns Monographie, gleichzeitig seine Habilitationsschrift (Rautmann 1921), deshalb in weiten Teilen als eine methodische Einführung in die Variabilitätsstatistik, also in die mathematischen Methoden zur Analyse von Unterschieden zwischen Individuen, insbesondere der Streu- 
ung von Ausprägungen einzelner Merkmale um einen Durchschnitt. In kurz nach dem Krieg entstandenen konstitutionspathologischen Lehrbüchern nehmen Kapitel zu grundlegenden statistischen Analyseverfahren ebenfalls großen Raum ein (Bauer 1921; Günther 1922).

Doch die Statistik konnte die Frage nach dem „normalen“ Körper - trotz vormals gegenteiliger Erwartungen - nicht alleine lösen. So verschärfte sich in der Folge die Diskussion um Abgrenzung und Bedeutung der „Norm“, was zu einer intensiven medizintheoretischen Debatte um Normund Krankheitsbegriff in den 1920er Jahren führte. Die beteiligten Konstitutionspathologen konnten dabei zu keiner Übereinkunft oder gar Lösung gelangen, aber die ausdifferenzierte Debatte arbeitete die bis heute gültigen Eckpunkte und Hauptfragen des Normproblems in der Medizin heraus.

Die rigorose Nutzung des Ersten Weltkrieges zur medizinischen Forschung ist bekannt (Eckart \& Gradmann 1996, Eckart 2014). Die Arbeiten von Livia Prüll (1996, 2003) etablieren, dass die von Ludwig Aschoff konzipierte großangelegte Kriegspathologie auch durch Konstitutionsforschung motiviert war. Das exzessive Sammeln von Daten durch die Kriegspathologie thematisiert außerdem Philipp Felsch (2005), dessen Aufsatz sich ähnlich wie der hier vorliegende mit Messen, Sammeln und Rechnen verschiedener medizinischer Disziplinen unter konstitutionsmedizinischer Fragestellung zwischen 1900 und 1930 beschäftigt. Felsch stellt seine Studie unter das Vorzeichen des Dualismus zwischen Ganzheitsanspruch und technischen Methoden, welche in der konstitutionellen Medizin produktiv vereint werden. Anders als Felsch fokussiere ich in meinem Aufsatz auf anthropometrische Untersuchungen in der Inneren Medizin sowie einen sehr engen, aber als Drehpunkt herausgearbeiteten Zeitraum von wenigen Jahren. Diese verdichtete Perspektive macht es möglich, nicht nur übergreifende Tendenzen, sondern konkret entscheidende Entwicklungen innerhalb der Konstitutionslehre und der Inneren Medizin betreffend die Aneignung von anthropometrischen wie mathematisch-statistischen Methoden und der Problematisierung des Normbegriffs nachzuvollziehen.

Die vorliegende Arbeit verortet sich in der rezenten Forschung zur Konstitutionslehre durch Carsten Timmermann (1996, 2001), Michael Hau (2000) und Nadine Metzger $(2016,2017){ }^{3}$ Die berührten Forschungsfelder Geschichte der medizinischen Anthropometrie, der mathematischen Statistik in der Medizin und des Normdiskurses sind bereits unterschiedlich stark von Medizin- und Wissenschaftsgeschichte bearbeitet worden. Sehr gut ist der Stand der Literatur zur Normdebatte der 1920er Jahre im Speziellen sowie dem medizinischen Normproblem im Allgemeinen, insbesondere auch dem hier relevanten Zusammenhang zwischen metrischen Methoden und Normierung. ${ }^{4}$ Nur wenige historiographische Arbeiten liegen hingegen für den Statistikgebrauch in der deutschsprachigen Medizin 
im Untersuchungszeitraum vor, wenn man von der etwas anders gelagerten Medizinalstatistik absieht. ${ }^{5}$ Literatur zu anthropometrischen Methoden findet man im Zusammenhang mit der Normdebatte, aber auch in Bezug auf Militärmedizin und Anthropologie. ${ }^{6}$ Anthropometrische Reihenmessungen durch konstitutionspathologisch motivierte Internisten während und kurz nach dem Ersten Weltkrieg haben in der Sekundärliteratur noch kaum Aufmerksamkeit erfahren. ${ }^{7}$

Der enge Zusammenhang zwischen der Einführung metrischer Methoden und einem reflektierten Normbegriff in den Lebenswissenschaften ist bereits durch die Forschung herausgearbeitet worden (siehe etwa Hess 1997). Die vorliegende Arbeit kann allerdings an ihrem Quellensample besonders gut die mehrschrittige Entwicklung herausarbeiten, die von ungeordnetem Datensammeln über Methodenreflexion bis hin zur medizintheoretischen Grundlagendebatte führte. Die behandelten Konstitutionspathologen erarbeiteten sich in den Jahren 1914 bis 1922 einen bemerkenswerten Zuwachs an einschlägiger Expertise in anthropometrischen und mathematisch-statistischen Methoden sowie medizintheoretischer Problematisierung, so dass an diesem Beispiel deutlich werden kann, wie und warum Arbeitsweisen, die heute für die medizinische Epistemologie zentral sind, implementiert und verbreitet wurden - selbst wenn es wie im Fall von mathematischer Statistik und dem Normalwertkonzept noch Jahrzehnte dauern sollte, bis sich diese in der Medizin vollständig etabliert haben sollten.

Im Folgenden werde ich zunächst das Forschungsprogramm der Konstitutionslehre vor und im Ersten Weltkrieg darstellen sowie ihren Aufruf zur Datensammlung herausarbeiten. Dann soll die These in den drei Schritten „Daten sammeln“ (Anthropometrie), „Daten auswerten“ (Statistik) und „Daten interpretieren“ (Normdebatte) dargelegt werden. Ein Fazit ordnet das Dargestellte ein in den Verlauf und die Wirkung der Konstitutionslehre über den Untersuchungszeitraum hinaus.

\section{Der (anthropometrische) Auftrag der Konstitutionslehre an den Arzt im Weltkrieg}

Erste Forderungen und Ansätze einer modernen Konstitutionslehre entstanden um 1900, als vereinzelte Stimmen den Blick auf Krankheitsanfälligkeit und Individualität des einzelnen Patienten lenkten - gegen das als dominant wahrgenommene Krankheitskonzept der Bakteriologie, dem vorgeworfen wurde, Krankheiten einseitig durch äußere Ursachen verursacht zu sehen. Statt dessen wurde die individuelle Konstitution betont, 
die Ausbruch und Verlauf von Krankheiten mindestens zu gleichen Teilen bestimme wie externe Krankheitserreger. Vertreter dieser Auffassung - Internisten, Pathologen, Hygieniker, Anatomen - suchten dabei genauso ihre eigenen Disziplinen gegenüber der Bakteriologie stark zu machen, wie sie sich gegen den Vorwurf wehren mussten, zur vormodernen humoralpathologischen Medizin zurückzukehren (Metzger 2016).

Für die Legitimation und Bedeutung über vereinzelte Vertreter hinaus erwies sich der Internistenkongress in Wiesbaden des Jahres 1911 als bedeutsam, der die Konstitution als Hauptthema des Kongresses verhandelte (Metzger 2016). Die Hauptredner entwarfen dabei vor dem wichtigsten und größten internistischen Forum der deutschsprachigen Länder ein Forschungsprogramm für die Konstitutionslehre der Zukunft (Krehl 1911; His 1911; Pfaundler 1911), da es noch kaum haltbare Erkenntnisse zum Thema gab. Gleichzeitig wurden große Hoffnungen in die zukünftige Konstitutionslehre gesetzt, durch ihre Grundlagenforschung sollte sie die Perspektive ärztlicher Theorie und Praxis (wieder) auf den einzelnen Patienten lenken und letztlich dadurch die individuelle Therapie verbessern. Die Redner bezogen sich dabei stark auf den Rostocker Internisten Friedrich Martius (1850-1923), der sich bereits vor 1900 für eine „Renaissance“ des Konstitutionsbegriffes eingesetzt habe (His 1911: 24). Drei Jahre später legte Martius (1914) dann eine systematische Ausarbeitung seiner Vorstellungen zu "Grundbegriffe[n] und [...] der möglichen Methodik“ der Konstitutionsforschung vor.

Diese in den Jahren vor dem Ersten Weltkrieg veröffentlichten Visionen einer zukünftigen Konstitutionslehre bestanden auf dezidiert naturwissenschaftlichen Methoden und forderten darüber hinaus eine epistemologische Modernisierung ihrer Fächer, sprich Ausrichtung an den Naturwissenschaften. Anders als die zeitgenössischen Leitdisziplinen Bakteriologie und Physiologie, die stark auf Experimente zum Erkenntnisgewinn ausgerichtet waren, hatte eine naturwissenschaftliche Orientierung die meisten der Fächer, die sich für die Konstitutionslehre engagierten, erst wenig erfasst. Gerade Internisten oder Vertreter verwandter Fächer (Pädiatrie, Dermatologie etc.) sahen deshalb im Hinblick auf eine reflektierte Methodik noch großes Potenzial für ihre Disziplinen. So forderte Martius bereits früh konstitutionspathologische Grundlagenforschung „auf Grund streng exacter naturwissenschaftlicher Beobachtung am Krankenbette und auf Grund streng exacter Experimente an Mensch und Tier" (Martius 1909[1900]: 185). Konstitutionsforschung "auf strengster wissenschaftlicher Grundlage“ (Krehl 1911: 11) wurde auf dem Internistenkongress in Wiesbaden verlangt, im ersten Band der neugegründeten Zeitschrift für angewandte Anatomie und Konstitutionslehre einige Jahre später „moderne exakte Forschung“ (Neuburger 1914: 10). 
Um diese Forderungen umzusetzen, setzte man auf metrische Verfahren und Statistik - zumal für Fächer, in denen das naturwissenschaftliche Experiment nicht anzuwenden war. Bereits Martius (1909[1900]: 188) hatte imaginiert, der Konstitutionslehre „auf rein statistischem Wege eine breitere und allgemein giltige [sic] Unterlage zu verschaffen." Den Wert einer solchen Unternehmung, zumal auf Grundlage von Daten aus der geringgeschätzten Versicherungsstatistik, musste Martius im Jahr 1900 noch ausführlich begründen (Martius 1909[1900]: 188-191) - ab 1911 war dann die Forderung nach einer breiten metrischen Datengrundlage in der Konstitutionslehre Standard. Auf dem Wiesbadener Kongress betonte Wilhelm His (1863-1934), dass Erkenntnis über Konstitution „nicht aus zufälligen Einzelbildern, sondern aus statistisch geordneten, gehäuften Krankheitsfällen“ (1911: 31) gewonnen werden müsse und für den Pathologen Otto Lubarsch (1860-1933) stand noch zehn Jahre später fest, dass man „ein möglichst großes in Zahl und Maß ausdrückbares Tatsachenmaterial herbeischaffen müsse[n], damit die Konstitutionslehre wissenschaftlich sicher begründet wird“ (1921: 816). Wissenschaftlichkeit und metrische Methoden wurden somit gleichgesetzt. ${ }^{8}$

In dieser frühen Phase der Konstitutionslehre herrschte somit bei der Methodenfrage weitgehend Einigkeit (Metzger 2016). Erst in den 1920er Jahren sollten durch den Einfluss des Holismus (Harrington 1999; Lawrence \& Weisz 1998) ganzheitlich ausgerichtete und intuitive Ansätze Eingang in die Konstitutionslehre finden und deren Methodenspektrum weiter auffächern (Metzger 2017). Uneins waren sich die Konstitutionspathologen hingegen durchgängig bei den Fragen, wie genau „Konstitution“ zu definieren sei und welche Rolle der Vererbung bei deren Ausprägung zukomme. Trotz einer Fülle verschiedener und konkurrierender Konstitutionsbegriffe musste jedoch für jeden Konstitutionspathologen die konkrete Arbeit am aktuellen Körperzustand der untersuchten Patienten ansetzen, so dass die Frage nach einem gesundhaften, „normalen“ Körper für alle von zentraler Bedeutung war.

Der Begriff der „normalen“ Konstitution beziehungsweise allgemein der „Norm“ bezog sich in unausgesprochener Übereinkunft dabei zumeist auf den männlichen Körper und wurde zunächst gänzlich vorreflexiv verwendet, ohne ihn näher zu problematisieren: Die „Norm“ entspräche einem "gesundhaften Durchschnitte“ (Martius 1909: 187) oder sei darin fassbar, „wie sie von der Mehrzahl der Menschen charakterisiert wird“ (His 1911: 26). Erst ab 1914 erkannten Konstitutionspathologen dies als Problem (Tandler 1914: 12) und versuchten sich an ersten Definitionen. Martius legte zunächst Wert darauf, dass pathologische Abweichungen von der Norm für den Menschen intuitiv erfassbar und damit unmittelbar eindeutig seien: „grobe Differenzen [...], bei denen es ohne weiteres offen- 
sichtlich ist, daß sie dem mittleren Durchschnitt vollkommen fremd sind“ (Martius 1914: 228). Der Anatom Julius Tandler (1869-1936), Herausgeber der Zeitschrift für angewandte Anatomie und Konstitutionslehre, definierte im gleichen Jahr die „normale Konstitution“ ebenfalls vage als das, was sich „durchschnittlich bewährt" habe, „also innerhalb der physiologischen Grenzen liegt" (1914: 14).

Doch auch für die zentrale Frage nach der normalen Konstitution verhieß das Sammeln metrischer Daten eine „solide Grundlage“. So nahm der Freiburger Internist Oskar de la Camp während des Weltkrieges an, dass die großen Mengen an Körpermaßen, die er und sein Mitarbeiter Rautmann an Fliegerrekruten sammelten, feste Aussagen zum durchschnittlichen gesunden Körperbau ermöglichen würden. Er versuchte mit Hilfe der so gewonnenen Durchschnittsmaße eine „Konstitutionsformel“ aufzustellen, mit deren Hilfe man für jeden (männlichen) Patienten bestimmen könne, in wie weit er von der normalen Konstitution abweiche (de la Camp 1918; dazu Metzger 2017: 281-282). Innerhalb der Medizin war die Idee, den Körperzustand mithilfe einer Formel objektiv bestimmen zu können, ursprünglich durch den militärischen Kontext der Tauglichkeitsbestimmung sowie durch die Versicherungsmedizin etabliert worden (Hartmann 2011: 132-139; Florschütz 1914: 82-83), bei denen offensichtlich finanzielle Ansprüche und Rechtssicherheit im Hintergrund standen.

Es liegt auf der Hand, dass in Fragen von Tauglichkeit, Dienstbeschädigung, Erwerbsunfähigkeit und folgenden Rentenansprüchen von den ärztlichen Gutachtern eindeutige und vergleichbare Urteile gefordert wurden, was die Verwendung metrischer Verfahren begünstigte. Mit Porter (1995) zu folgern, dass metrische Methoden Ärzten stets von außen oktroyiert worden seien, greift aber zu kurz. Nicht nur fokussierten die frühen Konstitutionspathologen bereits vor dem Weltkrieg auf Maß, Zahl und Statistik, um ihre Disziplinen mit „naturwissenschaftlichen Methoden“ in der innermedizinischen Fächerkonkurrenz gut aufzustellen, sondern man versprach sich von der Anthropometrie auch Verbesserungen in Diagnostik und Prognose sowie der Prophylaxe. Genau dieses Programm machte Max Berliner in seinem Aufsatz „Über die Bedeutung der Anthropometrie für die Klinik“ auch noch nach dem Krieg stark, als er den Wert der anthropometrischen Habitusbestimmung für die klinische Praxis herausstellte und die Daten seiner Reihenuntersuchungen exemplarisch sogleich im Zusammenhang mit diversen Krankheiten präsentierte (Berliner 1920b: 343-344, 348, 354-355). Der Kliniker müsse lediglich den kurzen Weg „bis zur Gewöhnung an die Zahlenbegriffe“ zurücklegen, um diese „objektive Weise“ der Patientenbeschreibung fruchtbar in der Praxis anwenden zu können (ebd. 348). 
Als kontingentes Geschehen bot der Weltkrieg der jungen Konstitutionslehre in ungeahntem Maße die Möglichkeit, im großen Stil Reihenuntersuchungen an Gesunden durchzuführen, um ihr Problem der „normalen Konstitution“ zu lösen. So betonte der Pathologe Ludwig Aschoff 1916, „die nie wiederkehrende Gelegenheit, über die normale und krankhafte Konstitution unseres Volkes im wehrhaften Alter Kenntnisse zu sammeln, [dürfe] von uns ebenso wenig wie von den Klinikern unbenutzt bleiben" (Aschoff 1916: 2). Und Internisten wie de la Camp, Rautmann oder Theodor Brugsch taten genau dies - verhießen doch anthropometrische Reihenuntersuchungen an Soldaten sehr leicht wissenschaftlichen Ertrag abzuwerfen. Als Vorsitzender einer Untersuchungskommission auf Tauglichkeit zum Flugdienst erhielt de la Camp für sich und seinen Mitarbeiter Rautmann Zugriff auf große Zahlen von Probanden, sicherlich ein nicht zu unterschätzender Anreiz für den forschungswilligen Konstitutionspathologen. Hermann Rautmann vermaß und untersuchte während dieser Tätigkeit zwischen 1917 und 1918 innerhalb etwa eines Jahres mindestens 1.864 Soldaten und verfasste auf dieser Datengrundlage sein methodisch einflussreiches Buch Untersuchungen über die Norm (Rautmann 1921). Theodor Brugsch aus der 2. Medizinischen Klinik der Charité erhob zwischen 1914 und 1916 umfassende Körpermaße an 1.560 Heeresfreiwilligen beziehungsweise Rekruten, ${ }^{9}$ woraus er ein eigenes Körpertypen-System ableitete (Brugsch 1917; Brugsch 1918), das er auch in späteren Veröffentlichungen weiter benutzte (etwa Brugsch 1922). Doch auch außerhalb des Kriegsdienstes führten Konstitutionspathologen Reihenmessungen durch: Brugschs Berliner Kollege Max Berliner untersuchte kurz nach dem Krieg einige Grundmaße einerseits an sechzig Knaben aus seiner poliklinischen Tätigkeit an der Charité (Berliner 1920a), andererseits an hundert erwachsenen männlichen Patienten (Berliner 1920b); eine dritte Messreihe zu den Thoraxmaßen von 126 Männern veröffentlichte er ein Jahr später (Berliner 1921).

Alle drei waren aufstrebende junge Internisten, die sich mit ihren anthropometrischen Arbeiten wissenschaftlich zu profilieren suchten: Hermann Rautmann (1885-1956) habilitierte sich 1921 und wurde seitdem als herausragender Vertreter einer anthropometrischen Konstitutionslehre wahrgenommen (Metzger 2020). Der etwas ältere Theodor Brugsch (1878-1963) sollte mit seiner „Personallehre“ in den 1920ern zu einem der wichtigsten Ideengeber der Konstitutionslehre werden (Metzger 2017; Gadebusch Bondio 2015: 24-37). Zu Max Berliner (1888-1960/65) bietet die medizinhistorische Literatur bislang noch keinen biographischen Abriss, lediglich kürzere Erwähnungen: Während seiner Zeit an der Kraus'schen Klinik der Charité arbeitete er unter anderem auch über Hoch- und Zwergwuchs und habilitierte sich 1925. Seit 1930 war er außerordentlicher Professor (Schagen 2008: 58). Zwischen 1927 und 1930 widmete er sich als exter- 
ner Mitarbeiter des Kaiser-Wilhelm-Instituts für Anthropologie, menschliche Erblehre und Eugenik der Blutgruppenforschung (Massin 2003: 197; Schmuhl 2005: 78; 96-97), außerdem war er am Berliner Institut für Krebsforschung tätig. Er war verheiratet mit Lili Orenstein (1891-1972), einer Tochter des Eisenbahnindustriellen Benno Orenstein (1851-1926) (Zabel 1999). Berliner war getauft, galt nach den Nürnberger Gesetzen der Nationalsozialisten aber als „jüdisch“ und wurde 1935 von der Hochschule vertrieben (Pawliczek 2011: 476; Schagen 2008: 58). Mit seiner Frau emigrierte er nach New York, wo er in Brooklyn eine Praxis betrieb (Ruppel 2009: 102; Zabel 1999). Er starb dort 1960 oder 1965 (Zabel 1999).

Anthropometrische Studien wurden im Untersuchungszeitraum natürlich auch außerhalb der Konstitutionspathologie durchgeführt, insbesondere zu Wachstums- und Ernährungsfragen: Einige Prominenz innerhalb der Methodendiskussion der Konstitutionsforscher erlangten die „KörpermaßStudien" an Münchner Schulkindern des Pädiaters Meinhard von Pfaundler (1872-1947) und seiner Mitarbeiter (Pfaundler 1916; Skibinski 1914; Riedel 1913; Dikanski 1914). Auf Ernährung fokussierten die Untersuchungen zum Normalgewicht durch den Internisten Gustav Oeder, Inhaber einer Diätkuranstalt bei Dresden (Oeder 1915a, b). Neu waren anthropometrische Erhebungen nicht - wie die umfassende und vielzitierte Sammlung medizinischer Daten des Tübinger Internisten Hermann Vierordt (1853-1943) zeigt, lagen umfassend Messdaten für äußere wie innere Körperteile vor, die seit den 1830er Jahren (verstärkt seit den 1880ern) hauptsächlich von Bevölkerungs- und Militärstatistikern, Anatomen und Anthropologen gesammelt worden waren (3. Aufl. 1906). Gynäkologen und Geburtshelfer hatten Daten für Neugeborene und Kinder vorgelegt. Die von Vierordt gesammelten Untersuchungen zielten allerdings ausschließlich darauf, Durchschnittswerte für ihre jeweiligen Untersuchungsgegenstände anzugeben, was den hier untersuchten Konstitutionsforschern nicht mehr genügte. Zudem räumte die Epistemologie der Konstitutionslehre während des Weltkrieges sowie kurz danach dem anthropometrischen und statistischen Zugriff besonderes Gewicht ein - sehr viel mehr als Vierordts internistische Leser dies zuvor getan hatten. Deutlich wird dieser Fokus sowohl in theoretischen Ausarbeitungen zur konstitutionspathologischen Anthropometrie (Martius 1914; Kraus 1917) als auch den ersten Lehrbüchern für Konstitutionslehre (Bauer 1921, 1923; Günther 1922). ${ }^{10}$ 


\section{Daten erheben: Konstitutionspathologische Anthropometrie}

Hermann Rautmann berichtete in seiner Monographie ausführlich über die praktischen Schwierigkeiten, die sich ihm im Frühherbst 1917 nach Übernahme der Fliegeruntersuchungskommission bei der Datenerhebung stellten. Diese Kommission prüfte die Tauglichkeit von Offizieren und Mannschaften, die sich aus anderen Truppenteilen freiwillig zum Flugdienst gemeldet hatten oder die bereits als Piloten oder Fliegerbeobachter tätig gewesen waren und über deren fortwährende Eignung entschieden werden sollte (Rautmann 1921: 18). Neben Körpermaßen wurden Seh-, Hörund Gleichgewichtsfähigkeit erhoben, Kreislauf und Atemapparat untersucht sowie neurologische und psychologische Tests im Hinblick auf den Flugdienst durchgeführt. Als doppelt promovierter Mediziner und Naturwissenschaftler deckte Rautmann schnell diverse Fehlerquellen auf, die die Messdaten wissenschaftlich unbrauchbar machten. So wurden die Soldaten zunächst lediglich nach ihrer Körpergröße befragt und nur nachgemessen, wenn der Betreffende diese nicht angeben konnte. Häufig wurden Körpergröße und Körpergewicht in bekleidetem Zustand inklusive Stiefeln erhoben. Schließlich verfügte Rautmann, „grundsätzlich bei jedem die Körpergröße ohne Stiefel und Strümpfe“ und das „Nacktgewicht mit einer Dezimalwaage“ zu erheben, um sicher vergleichbare Daten zu gewinnen (Rautmann 1921: 19-20). Als Wissenschaftler untersuchte er daraufhin, wie stark die Angaben variierten: Befragte man die Männer lediglich nach ihrer Körpergröße, wichen die Angaben bis zu $5 \mathrm{~cm}$ vom gemessenen Wert ab. Stiefel und Socken erzeugten zwischen 2 und $4 \mathrm{~cm}$ mehr an Körpergröße. Auch das Gewicht der Kleider variierte so stark, dass man es nicht einfach subtrahieren konnte. Neben diesen prozeduralen Faktoren identifizierte Rautmann auch eine menschliche Fehlerquelle: Der ,junge Hilfsdienstpflichtige“, der die Messungen vornahm, arbeitete ungenau, so dass Rautmann ihn durch einen hilfsdienstpflichtigen Architekten ersetzte, der die Messungen „sehr gewissenhaft und mit Verständnis“ durchführte (Rautmann 1921: 20).

Bei der Bestimmung des Blutdrucks erprobte Rautmann verschiedene Messgeräte, einmal den "kleinen Tonometer von Recklinghausen“ und daneben den "Hg.-Manometer von Riva-Rocci“ sowie andere QuecksilberManometer, wobei sich das Recklinghausen'sche Gerät als wenig zuverlässig erwies (Rautmann 1921: 21). Doch er hatte nicht nur mit solchen technischen Herausforderungen zu kämpfen - da es noch keine Übereinkunft zur standardisierten Einheit gab, in der Blutdruckwerte angegeben werden sollten, arbeiteten die beiden von Rautmann benutzten Geräte mit verschiedenen Angaben für den systolischen Blutdruck, die jeweils umgerechnet werden mussten (Rautmann 1921: 22). Zudem stand beim Blut- 
druck noch nicht fest, was die so gewonnenen Daten überhaupt aussagten - man befand sich noch mitten im Prozess der Standardisierung und klinischen Relevanzsuche (Groedel 1919; Fangerau \& Martin 2014: 82-85). Ähnlich verhielt es sich mit dem Querdurchmesser des Herzens, den Rautmann mit Hilfe eines „Groedelschen Orthodiagraphen“ zu bestimmen suchte, der eine röntgenologische Aufnahme des Herzschattens machte (Rautmann 1921: 21). Ziel war es hier, Normalmaße in Relation zur Körpergröße zu gewinnen. Ob ein vergrößertes Herz, zum Beispiel bei einem Sportler, nun pathologisch sei oder nicht, war eine kontrovers diskutierte offene Forschungsfrage (Metzger 2020), deren vorherige Klärung für die Interpretation von Rautmanns Messergebnissen essentiell war.

Durch seine ausführliche Beschreibung der Methoden gab Rautmann in seinem Buch somit auch Einblicke, wie unpräzise und unreflektiert anthropometrische Messungen bei Musterungen ablaufen konnten, wenn kein wissenschaftlich interessierter Arzt den Messenden über die Schultern schaute. Allerdings gab es auch offizielle Vorschriften des Militärsanitätsdienstes, wie gemessen werden sollte, damit vergleichbare Ergebnisse produziert wurden - die Vorgaben wurden allerdings häufig nicht eingehalten (Hartmann 2011: 139-41). Besonders augenfällig ist die Notwendigkeit von Messvorschriften bei der Erhebung des Brustumfangs, der als ein Indikator für Leistungsfähigkeit galt. Seit den 1860er Jahren war die Vergleichbarkeit von Brustumfangsmaßen von Militärärzten ausführlich problematisiert worden (Hartmann 2011: 132-134); den Dienstanweisungen des Militärsanitätsdienstes des Ersten Weltkrieges lag das Verfahren des Generalarztes Franz Hermann Frölich (1839-1900) zugrunde, das dieser 1872 aufgestellt hatte (Pagel 1901: 561; Frölich 1872). Dabei wurde mit „seitwärts bis zur Wagerechten“ erhobenen Armen auf der Höhe der unteren Schulterblattwinkel beziehungsweise knapp unter den Brustwarzen bei ruhiger Atmung gemessen, danach bei „möglichst tiefer Ein- und Ausatmung“, so Rautmann (1921: 21) mit Verweis auf die relevante militärärztliche Literatur.

Brugsch und Berliner, die in ihren Reihenmessungen ebenfalls den Brustumfang erhoben, lassen solchen reflektierten Umgang mit dem Problem hingegen vermissen. Theodor Brugsch ging in seinem Aufsatz (1917) insgesamt kaum auf Messmethoden ein und spezifizierte vor allem auch die Methode nicht eindeutig, mit der er oder seine Mitarbeiter den Brustumfang gemessen hatten. Zwar könnte man annehmen, dass er sich an die militärische Dienstanweisung gehalten habe, zumal Brugsch diese auch in einem etwa zeitgleich erschienenen Handbuch beschrieb (Brugsch \& Schittenhelm 1918: 49), aber allein das Versäumnis, dies explizit zu äußern, wirft ein bezeichnendes Licht auf das wissenschaftliche Niveau seiner Untersuchung, auf die er nicht nur sein Körpertypensystem auf- 
baute, sondern auf deren Ergebnisse er in der Zukunft auch immer wieder als Normwerte rekurrieren würde. Erschwerend kommt hinzu, dass sich Brugsch für Vergleichswerte und Messschema auf den amerikanischen Astronomen Benjamin Apthorp Gould (1824-1896) bezog, der während des Sezessionskrieges militärärztliche Vermessungen an über 10.000 Soldaten durchgeführt hatte (Gould 1869; Brugsch 1917: 5). Goulds Daten basierten allerdings auf sehr ungenauen Messungen, zum Beispiel wurde der Brustumfang am bekleideten Soldaten unter dem Hemd etwa auf Höhe der Brustwarzen gemessen, also ohne vollständigem Sichtkontakt (Gould 1869: 239; 262-263). Dies schränkte die Aussagekraft der Daten sehr ein, was Brugsch bei gründlicherer Lektüre von Gould (1869) hätte auffallen müssen.

Auch sein Charité-Kollege Berliner (1920a, b) machte die Messmethoden seiner verschiedenen Erhebungen nicht explizit, obwohl er seine Ergebnisse mit denen anderer Autoren verglich (1920a: 34). Da er jedoch in einer seiner drei Veröffentlichungen die militärische Messmethode als einzige ausführlich beschrieb, ist anzunehmen, dass er diese auch benutzt hatte (1920b: 346).

Keiner der drei internistischen Konstitutionspathologen stützte sich somit auf die zeitgenössische Anthropologie, die bereits sehr ausführliche Messanleitungen für die Anthropometrie produziert hatte (Martin 1914). So überrascht es nicht, dass deutschsprachige Anthropologen den männlichen Brustumfang anders bestimmten: Rudolf Martin (1864-1926) setzte das Maßband höher an, und zwar oberhalb der Brustwarzen (Martin 1914: 149-50; Martin 1924: 29); die genaue Position der Arme während des Messens befand sich allerdings noch unter Diskussion (Martin 1914: 149-150; vs. Martin 1922: 387; und Martin 1924: 29). Zu Martins ausgefeilten Messmethoden gehörte auch ein spezielles Instrumenteset, das kostspielig angeschafft werden musste (Martin 1914, 1922). International hatten sich die Anthropologen und Prähistorischen Archäologen im Jahr 1912 wiederum auf ein anderes Brustmessverfahren geeinigt, das das Maßband weitaus tiefer anlegte, auf der Höhe des Processus xiphoideus am unteren Ende des Brustbeins, aber in Deutschland seltener rezipiert wurde (Scheidt 1921: 58). Die Messmethoden von Rudolf Martin hingegen verbreiteten sich bald schon über die Anthropologie hinaus (Morris-Reich 2013), und die Konstitutionslehre rekurrierte ab den frühen 1920er Jahren fast durchgängig auf dessen „Vorschriften“ zur Anthropometrie (vgl. Kraus 1917: 259; Günther 1922: 63; Borchardt 1924: 293).

Es ist auffällig, dass Brugsch (1917), Rautmann (1921) und Berliner (1920a, b) als konstitutionspathologische Pioniere in der Körpervermessung in den ersten Veröffentlichungen ihrer Reihenmessungen (noch) kaum anthropologische Literatur zitierten. Als Brugsch allerdings ein Jahr 
später den ursprünglichen Aufsatz für seine Monographie Allgemeine Prognostik leicht umarbeitete, verwies er auf die dritte Auflage von Der Mensch des Münchener Anthropologen Johannes Ranke (1836-1916) (Brugsch 1918: 44; Ranke 1912). Ranke, der seit 1886 an der Philosophischen Fakultät München den ersten deutschen Lehrstuhl für Anthropologie bekleidete (Hoßfeld 2005: 171-172), hatte die erste Auflage dieses Werkes bereits 1886/87 verfasst, so dass sich hier der Eindruck wiederholt, dass Brugsch sich bei anthropometrischen Fragen an jahrzehntealter Literatur orientierte, anstatt aktuellere Forschung auszuwerten.

Andere frühe Konstitutionspathologen rezipierten sehr wohl anthropologische Literatur als sie sich auf das Feld der Anthropometrie vorwagten: So zitierten Pfaundler und seine Doktoranden in den „Körpermaß-Studien" an Münchener Schulkindern auch anthropologische Autoren neben Bevölkerungsstatistikern für Vergleichsdaten (Pfaundler 1916; Riedel 1913; Skibinski 1914; Dikanski 1914). Die Münchener Studie verortete sich allerdings nicht in der Konstitutionslehre und gewann ihre beiden Maße, Körpergröße und -gewicht, aus bereits vorhandenen Schulakten. Friedrich Kraus (1858-1936), Direktor der 2. Medizinischen Klinik der Charité und damit Vorgesetzter von Brugsch und Berliner, sprach im Rahmen einer Fortbildungsveranstaltung zu militärärztlicher Sachverständigentätigkeit Ende des Jahres 1916 über konstitutionspathologische Begutachtung mithilfe von anthropometrischer Messung und empfahl dabei ausdrücklich Martins Lehrbuch für die Messmethoden, verwies beim Thema Brustumfang aber auf die verbindlichen Regelungen der Kriegsmusterungsanleitung von 1916 (Kraus 1917: 259, 62). Kraus führte selbst keine Messungen durch, aber sein Vortrag lässt erkennen, dass auch für ihn die Anthropometrie zentral für eine objektive Feststellung der körperlichen Konstitution war.

Auch wenn Brugsch, Rautmann und Berliner es zunächst versäumten, anthropologische Messmethoden zu rezipieren, setzten sich diese in ihren späteren Veröffentlichungen durch. Rautmann (1924: 567) und Brugsch (1922: 60) verwiesen dabei jeweils auf Martins Methodenkatalog, Brugsch hingegen versuchte, seine eigene internistische Version zu verbreiten (Brugsch 1922; Berliner et al. 1923). In den verschiedenen Auflagen des von Brugsch herausgegebenen Lehrbuch klinischer Untersuchungsmethoden wechseln die Vorschriften zur Thoraxmessung dementsprechend auch kurz nach dem Krieg von der militärischen (Brugsch \& Schittenhelm 1918: 49) zu Martins anthropologischer Methode (Berliner et al. 1923: 68). Während des Krieges scheinen gerade Brugsch und Rautmann als Militärärzte die sich bietenden Möglichkeiten zu Reihenmessungen schnell genutzt und sich ohne große Vorbereitung in deren Durchführung gestürzt zu haben. Vorbereitende Lektüre der relevanten Forschungsliteratur sowie eine reflektierte Versuchsplanung vor der Durchführung blieben 
auf der Strecke oder wurden wie im Fall von Rautmann nachträglich implementiert.

\section{Daten auswerten: Konstitutionslehre und medizinische Statistik}

„Die in der Medizin meist gebräuchliche Beschreibung von Variationen beschränkt sich auf Angabe des Mittelwerts“, beklagte Pfaundler (1916: 10). Dies müsse sich dringend ändern, „damit Vertreter anderer Fächer nicht weiterhin Anlaß haben, über die unsachgemäße Behandlung solcher Dinge [...] der Mediziner zu lächeln“ (ebd.). Gleiches hatte der Anatom und Anthropologe Ludwig Stieda (1837-1918) bereits 1883 für seine Profession festgestellt (1883: 168) - es scheint sich also in den dreißig Jahren dazwischen nicht viel geändert zu haben. Auch Berliner, Brugsch und Rautmann, die zwischen 1917 und 1922 stark an Anthropometrie und mathematischstatistischen Methoden arbeiteten, setzten noch am gleichen Punkt an: Variationsbreite statt Mittelwerte.

Tatsächlich arbeitete die klinische Medizin dieser Zeit noch hauptsächlich mit Mittelwerten, die mit Normwerten gleichgesetzt wurden, wie das umfangreiche Nachschlagewerk von Hermann Vierordt (1906) zeigt, in dem anatomische, physiologische und physikalische Mittelwerte aus unzähligen Einzelstudien zusammengetragen wurden, um Ärzten Referenzwerte zu liefern. Doch Mittelwerte suggerieren einen physiologischen Idealpunkt, dem die meisten Menschen nicht entsprechen könnten, ohne dass dies pathologisch sei, kritisierte bereits Martius. Die Konstitutionslehre fokussiere hingegen auf die körperlichen Unterschiede zwischen Menschen, ihre jeweilige „persönliche Konstitution“, und damit die Varianz (1914: 74-76).

In diesem Sinne bemühten sich Berliner, Brugsch und Rautmann die Variationsbreiten der von ihnen anthropometrisch untersuchten Körpermerkmale in Zahlen zu fassen. Im einfachsten Fall konnte dies dadurch erfolgen, dass neben dem arithmetischen Mittel auch Minimum und Maximum der beobachteten Werte in absoluten Zahlen aufgeführt wurden, wie dies der statistisch weitgehend ahnungslose Max Berliner tat (Berliner 1920a, b, 1921). Theodor Brugsch benutzte in seinen ersten Arbeiten Balkendiagramme (Abb. 1) und das ein oder andere Kurvendiagramm als eingängige Hilfsmittel, um Variationsbreiten zu veranschaulichen, kaum jedoch mathematische Ausdrucksmittel (Brugsch 1917, 1918). Bemerkenswert ist, wie naiv manche Konstitutionsforscher dabei mit Zahlen umgingen. Gerade Max Berliner machte simple logische Fehler; zum Beispiel verglich er Unterschiede zwischen zwei Zahlenreihen von höchst unter- 


\section{Kurve 1.}

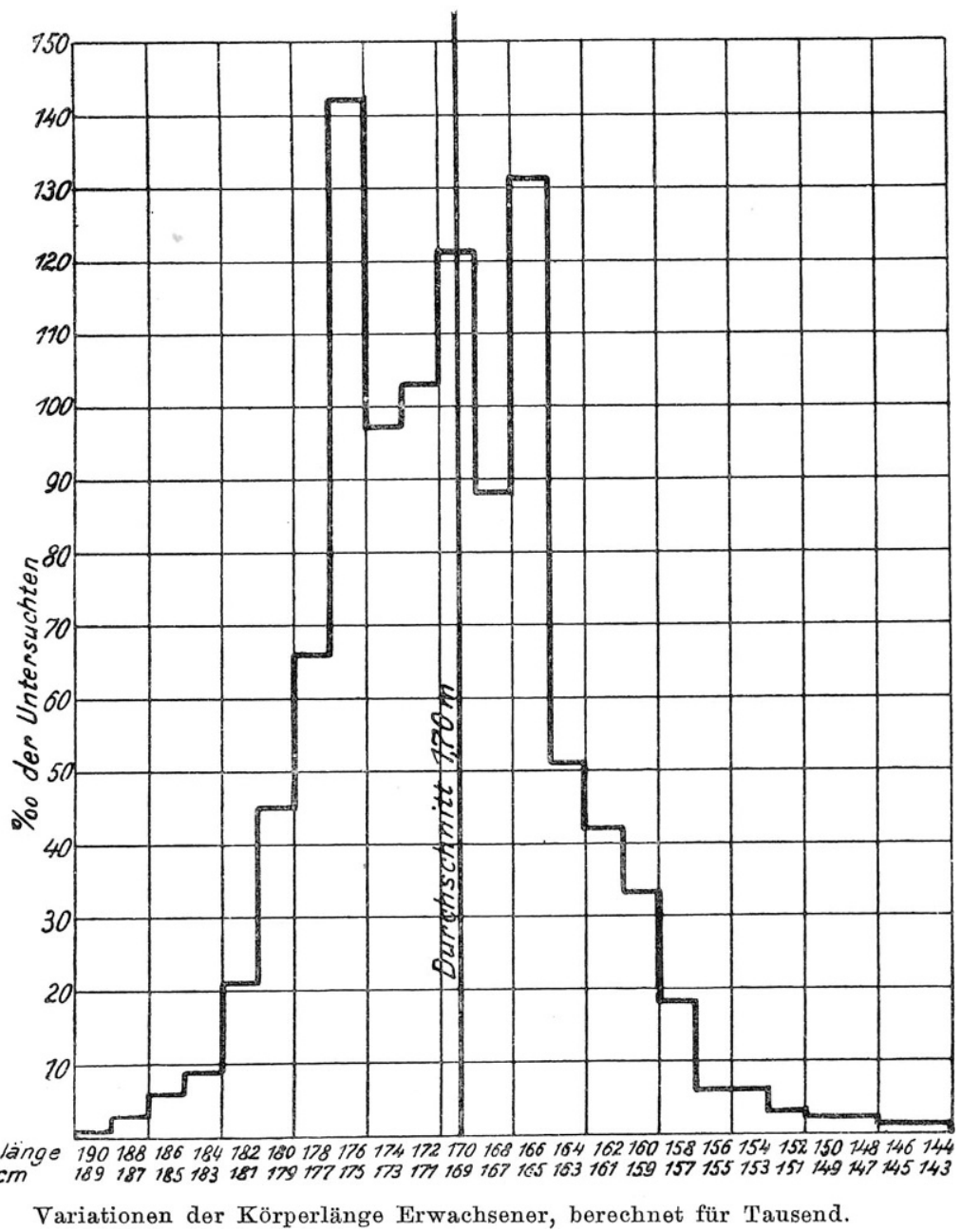

Abb. 1 Balkendiagramm zur Variabilität der Körpergröße bei Brugsch (1918: 27)

schiedlichen Dimensionen in absoluten Zahlen (Berliner 1920b: 349). Der statistische Kenntnisstand war offenbar noch so gering, dass dies ein adäquates Forschungsniveau unter Internisten darstellte.

Hermann Rautmann hingegen wählte explizit die Variabilitätsstatistik als Methode und erklärte, dass „die Parameter der [Gauß'schen] Verteilungskurve zusammen mit ihrem zugehörigen Richtwert eine Variationsreihe zutreffend und vollständig kennzeichnen können, nicht aber die Extremwerte, und daß hierzu natürlich auch die Durchschnittswerte allein ungenügend" seien (Rautmann 1921: 69). Rautmanns Buch verstand 
sich unter anderem auch als praktische Einführung in die statistischen Methoden der Kollektivmasslehre (1897) von Gustav Theodor Fechner (1801-1887), deren Anwendung er wärmstens empfahl. In den ersten Lehrbüchern für Konstitutionslehre von Julius Bauer (1887-1979) und Hans Günther (1884-1956) aus den Jahren 1921 und 1922 nahm dann die Variabilitätsstatistik eine zentrale Rolle ein: Bauer stellte sie an den Anfang seiner Vorlesungen über allgemeine Konstitutions- und Vererbungslehre gleich nach "Wesen und Ziele der Konstitutionspathologie" (Bauer 1921: 10-25), während Günther ihr gleich zwei von insgesamt elf Kapiteln widmete (Günther 1922: 35-63). Beide Autoren präsentierten eine grundlegende Einführung in statistische Analysemittel, die von Lesern ohne jegliche Vorkenntnis ausging.

Je mehr statistische Inhalte ihre Veröffentlichungen auswiesen, desto stärker sahen sich die Autoren in der Vorreiterrolle, diese Methoden in ihrer Disziplin zu verbreiten. Die Bücher von Rautmann, Bauer und Günther wollten ihren Lesern dezidiert dazu Anleitung bieten, aber auch Brugschs Veröffentlichungen sollten die Konstitutionslehre methodisch voranbringen. Umso eklatanter fällt daher ins Auge, dass die Autoren teilweise in grober Unkenntnis des mathematisch-statistischen Forschungsstandes waren. Dies betrifft zum einen die weit entwickelte englischsprachige Statistik - sichtbar etwa an der mangelnden Rezeption des englischen Mathematikers Karl Pearson (1857-1936), dessen Arbeiten um die Jahrhundertwende für die Entwicklung der modernen Statistik zentral waren („practically the founder of mathematical statistics", Porter 1995: 20). So formulierte Pearson in den 1890er Jahren den noch heute gebräuchlichen Korrelationskoeffizienten, mit dem der (lineare) Zusammenhang zwischen zwei Merkmalen mathematisch geprüft werden kann: Ein eigentlich für die Konstitutionsforschung attraktives Verfahren, da diese dezidiert nach Zusammenhängen zwischen Körpermerkmalen und Krankheitsneigungen suchte. Trotzdem wird in den frühen konstitutionspathologischen Veröffentlichungen der Korrelationskoeffizient nicht erwähnt. Zum anderen bewiesen Berliner und Brugsch zusätzlich große Lücken in der Rezeption der deutschsprachigen mathematischen Statistik, die in zum Teil auch für Anfänger gut verständlichen Einführungen leicht zugänglich gewesen wäre (Fechner 1897; Bruns 1906; Czuber 1908; Gruber \& Rüdin 1911; evtl. auch noch Kisskalt 1919) und durch ihre Verwendung in Anthropologie, Versicherungsmedizin und Vererbungslehre auch fachlich nahegelegen hätte.

Brugsch $(1917,1918)$ und Berliner $(1920 a, b, 1921)$ zitierten etwa bemerkenswert wenig (neuere) einschlägige Literatur, was besonders im Vergleich zu Rautmann (1921) auffällt. Ihr Fokus lag auf internistischer Literatur; eine Rezeption der Bevölkerungsstatistik bot sich nur wenig an, da das spezifische Problem der Variabilität biologischer Eigenschaften dort kaum 
interessierte (Žižek 1923: 8-12). Brugschs und Berliners Expertise zur Auswertung der Vermessung scheint fast ganz auf Adolphe Quetelets Klassiker (1838) und Pfaundlers „Körpermaß-Studien an Kindern“ (1916) zu ruhen. Zwar war letztere erst wenige Jahre alt und stellte auch die Gauß'sche Normalverteilung knapp vor, aber da sie hauptsächlich darauf abzielte, die Forschungsergebnisse mehrerer Doktoranden zusammenzufassen und zu kontextualisieren, waren hier die methodischen und vor allem auch statistischen Details dieser Dissertationen (Riedel 1913; Skibinski 1914; Dikanski 1914) nicht zu finden.

Rautmann zitierte neben seiner Hauptquelle (Fechner 1897) bemerkenswert viel statistische Fachliteratur von Autoren ganz verschiedener Professionen, darunter den Astronomen Heinrich Bruns, den Anthropologen und Mediziner Karl Ernst Greiner (Ranke \& Greiner 1904), den Philosophen Wilhelm Windelband, den Nationalökonomen Wilhelm Lexis und den dänischen Vererbungsbiologen Wilhelm Johannsen. Am Rande ging er auch auf Pearson ein, dem er im Gegensatz zu anderen deutsche Autoren eher positiv gegenüberstand (Rautmann 1921: 103n2). ${ }^{11}$ Die Rezeption Pearsons bot sich für Anthropologen und Konstitutionspathologen eigentlich auch deshalb an, da dieser anwendungsbezogen ebenfalls an anthropometrischen Fragestellungen arbeitete. Allerdings setzten sich seine heute maßgeblichen Konzepte in Deutschland nur äußerst stockend durch, was auch durch das Überlegenheitsgefühl der deutschen Wissenschaftler und nationale Animositäten begründet war (Bäumler 2010; vgl. Tröhler 1992: 72-74). Es überrascht daher nicht, dass sich Rautmann in seiner Studie auf Verfahren aus der deutschsprachigen Tradition beschränkte, zumal es sich um eine Qualifikationsarbeit an der nationalkonservativ dominierten Freiburger Fakultät (Hofer 2002: 67-73) handelte.

Über den dänischen Vererbungsforscher Wilhelm Johannsen (18571927) fanden Pearson und der Korrelationskoeffizient jedoch Eingang in die konstitutionspathologischen Einführungen von Julius Bauer (1921) und Hans Günther (1922). Johannsens Methodenlehrbuch, Elemente der exakten Erblichkeitslehre (1909), orientierte sich explizit an der „Pearson'schen Forschungsweise“ (Johannsen 1909: 494) und brachte dadurch die zeitgenössische englischsprachige Statistik in die deutsche Vererbungsforschung ein. Dies galt auch für die beiden anderen Einführungen in statistischmathematische Methoden, die von Bauer und Günther in ihren Statistikkapiteln zitiert wurden, die des Zoologen Arnold Lang (1914) sowie jene der Rassenhygieniker von Gruber und Rüdin (1911), wie sie im Führer der Gruppe „Rassenhygiene“ zur Internationalen Hygiene-Ausstellung in Dresden 1911 erschienen war. Sowohl Bauer als auch Günther scheinen Aufbau und Inhalte ihrer Kapitel stark an von Gruber und Rüdins leicht verständlicher Einführung in die Variabilitätsstatistik ausgerichtet zu haben; gerade 
die Internationale Hygiene-Ausstellung 1911 trug dazu bei, das Thema Statistik erfolgreich zu popularisieren (Nikolow 2001). Die Lehrbücher bauten damit auf einer ganz anderen statistischen Tradition als Rautmann und Brugsch auf - die meisten von Rautmann zitierten Autoren sind bei Bauer nicht zu finden. Günther ist der Erste, der sich zusätzlich auch mit seinen Vorgängern Brugsch, Rautmann und Bauer auseinandersetzte.

An den konstitutionspathologischen Autoren lässt sich nachverfolgen, dass sie nach ihren ersten Veröffentlichungen zur mathematischen Beschreibung biologischer Variabilität sehr schnell weitere statistische Kenntnisse erwarben. So veröffentlichte etwa Julius Bauer 1918 eine Studie zum Zusammenhang zwischen Körpertyp und Krankheitsneigung, für die er gut zweitausend Patienten seiner Poliklinik untersucht hatte und deren Ergebnisse er ausschließlich in Tabellen präsentierte, in denen die Häufigkeiten von Typen, Krankheiten und deren Kombinationen in Prozent angegeben wurden. Darüber hinaus erfolgte keine mathematische Analyse der Daten und es wurde keinerlei methodische Literatur zitiert, obschon sich die Arbeit als „statistische Forschung" verstand (Bauer 1918: 220). Drei Jahre später erklärte Bauer in seinem Lehrbuch dann Studierenden und interessierten Ärzten, wie man bei einer solchen Erhebung den „Korrelationskoeffizienten zweier alternativ variierender Merkmale oder Eigenschaften“ berechnete (Bauer 1921: 24-25). Auch bei Brugsch lässt sich eine schrittweise Zunahme statistischer Kenntnisse beobachten. Anders als in seinen frühen Veröffentlichungen verwies Brugsch 1922 in der zweiten Auflage der Allgemeinen Prognostik auf die Einführung von Johannsen und einige Grundbegriffe der Variabilitätsstatistik, ohne diese jedoch anzuwenden (Brugsch 1922: 58). Vier Jahre später platzierte er im von ihm herausgegebenen vierbändigen Kompendium Die Biologie der Person die Einführung in die Statistik prominent am Anfang des ersten Bandes (Salinger 1926). Rautmann (1921) verwendete zwar noch keinen Korrelationskoeffizienten und benutzte Begriffe aus der deutschsprachigen Tradition, spielte aber im Laufe der 1920er Jahre eine zentrale Rolle für die Förderung statistisch-mathematischer Methoden in Konstitutionslehre und Sportmedizin (Metzger 2020; Bäumler 2010: 80). 1923, also nur zwei Jahre nach der Veröffentlichung seiner Habilitationsschrift, warb Rautmann in der Münchener Medizinischen Wochenschrift für die Variabilitätsstatistik, empfahl die Benutzung des Pearson'schen Korrelationskoeffizienten und zitierte diverse englischsprachige statistische Literatur (Rautmann 1923). Für die Auswertung seiner nächsten Reihenuntersuchung verwies Rautmann dann für die statistischen Grundlagen auf Johannsen und benutzte wie dieser Bezeichnungen aus der englischsprachigen Tradition, zum Beispiel das noch heute gebräuchliche „ $\sigma^{“}$ für die Standardabweichung, einem wichtigen Maß für 
die Streuung eines Merkmals in einer Verteilung, nicht mehr das deutschsprachige „e“ (Rautmann 1924).

Diese Beispiele zeigen, dass sich die genannten Internisten innerhalb weniger Jahre grundlegende statistisch-mathematische Kenntnisse erschlossen und trotz einiger Verspätung noch Anschluss an die englischsprachige Statistiktradition fanden. Sie etablierten zudem einen Fachdiskurs zum Thema innerhalb der Konstitutionslehre, so dass insgesamt das Niveau konstitutionpathologischer Statistik bis 1925 sehr stark anstieg. Mitte der 1920er Jahre war die Variabilitätsstatistik dann schon so gut etabliert, dass die alleinige Angabe von Durchschnittswerten nicht mehr akzeptabel war und kein konstitutionspathologisches Lehrbuch ohne Statistik-Einführung auskam.

\section{Daten interpretieren: Die Diskussion um den Begriff der Norm}

Ein Jahr nach dem Erscheinen von Rautmanns Studie würdigte der Münchner Hygieniker und Sozialmediziner Ignaz Kaup (1970-1944) die anthropometrischen Reihenuntersuchungen von Brugsch und Rautmann als die „zwei bedeutendsten Studien über die Norm in den letzten Jahren“. Ihre „Absicht, für den Begriff des Normaltypus [...] feste Anhaltspunkte in Mass, Zahl und Gewicht zu finden, deutet die Hauptaufgabe moderner Konstitutionsforschung an“ (Kaup 1922: 162). Kaups offenkundiger Forschungsoptimismus in Bezug auf eine Festlegung des „Normaltypus“ sollte allerdings schnell durch eine Debatte unter Konstitutionspathologen gedämpft werden, die etwa zeitgleich Fahrt aufnahm: Die Diskussion um Definition, Anwendbarkeit und Bezugsrahmen des Begriffs der „Norm“ oder des „Normalen“.

Unabhängig von einander hatten 1921 drei Konstitutionspathologen auf verschiedene Probleme bei einer Normdefinition aufmerksam gemacht (Rautmann 1921: ix-3, 52-58; Bauer 1921: 107-109; Grote 1921: 6-12). Meinte „Norm“ einen Idealzustand oder den Durchschnitt? Sei „normal“ durch einen Mittelwert beschreibbar oder brauche es eine Variationsbreite? Wie breit sei letztere anzusetzen? Auf was beziehen sich solche Abgrenzungsversuche - auf die Menschheit, die Rasse, die Population, den Typus oder könne man lediglich am Individuum zu sich selbst Abweichungen feststellen? Fokussiere man auf physische Messgrößen am Körper, Morphologie oder Funktionstüchtigkeit von Organen? Anders als bei früheren Konstitutionspathologen war nun klar: „Eine rein logische Ableitung“ könne das Normproblem nicht lösen, man war „auf eine Vereinbarung angewiesen“ (Günther 1922: 51). Eine „natürliche“, dem Betrachter offenkundige 
Normabgrenzung, wie sie noch Martius (1914: 228) imaginiert hatte, war damit vom Tisch.

In den fünf Jahren bis 1926 erschienen weit über ein Dutzend Beiträge zum Thema, allein sieben in der Zeitschrift für Konstitutionslehre. In den Lehr- und Handbüchern der Konstitutionslehre wurde dem Normproblem zunehmend Raum gegeben: Bauer (1921: 107-109) problematisierte den Normbegriff auf wenigen Seiten, Günther (1922: 48-63) widmete der Debatte bereits ein ganzes Kapitel. In Brugschs Handbuch Biologie der Person fasste dann Kaup (1926) selbst den Stand der lebhaft geführten Diskussion in einem eigenen Kapitel zusammen, prominent recht weit vorne im ersten Band platziert. Die Normdebatte war zu einem zentralen medizintheoretischen Thema der Konstitutionslehre geworden (Borchardt 1924: v; Borchardt 1930: 16-31 mit Lit.).

Die bereits besprochenen Autoren schlugen erwartungsgemäß auch eine statistische Definition für eine Variationsbreite des Normalen vor: Rautmann (1921) und Bauer (1921) stützten sich dabei unabhängig voneinander auf die Standardabweichung einer Verteilungskurve und damit eine mathematisch definierte Größe: Diese entspricht bei der Gauß'schen Normalverteilung der Entfernung zwischen Mittelwert und einem der Wendepunkte der Kurve. Allerdings verlief nach der Empfehlung Bauers die Grenze zwischen dem Normalen und dem Abnormen bei der zweifachen

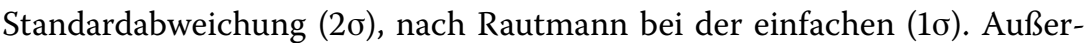
dem definierte Rautmann verschiedene Grade von Abnormität mithilfe der Normalverteilungskurve, etwa lediglich „abnorme“ und gar „krankhafte" Varianten (Rautmann 1921: 70-72). Günther zog die Grenze anhand der sogenannten Galton'schen Ogiven-Kurve, einer alternativen, heute wenig gebrauchten Beschreibungsmöglichkeit einer Verteilung, und legte fest, dass 95 Prozent aller Fälle als „normal“ gelten konnten - im Vergleich zu den 95,5 Prozent von Bauer und den 68,3 Prozent von Rautmann (Günther 1922: 58).

Auch die bereits während des Ersten Weltkrieges viel diskutierten Körpermaßindices wurden im Rahmen der Normdebatte stark in Frage gestellt. War es wirklich möglich, eine „normale“ Konstitution durch eine Körpermaßformel zu ermitteln, suggerierte diese doch eine gewisse Naturgesetzlichkeit der Größenverhältnisse am Körper? Verschiedene führende Konstitutionspathologen verabschiedeten sich von diesem Konzept, etwa Julius Bauer (Metzger 2017: 290), andere wie Kaup (1926) bewarben weiterhin ihre eigenen Formeln. Besonders radikal wandte sich Louis R. Grote (1886-1960) gegen schematische und reduktionistische Normkonzepte. Bereits in seinem initialen Beitrag sprach er sich für einen ausschließlich auf das Individuum bezogenen Normbegriff aus; klinisch sinnvoll sei lediglich, die Einzelperson mit sich selbst zu vergleichen, um zwischen ei- 
nem krankhaften und einem „normalen“ Zustand zu unterscheiden (Grote 1921: 7-12). Grotes Beitrag wurde kontrovers diskutiert, stand er doch der Variabilitätsstatistik diametral entgegen, während er ganzheitlich orientierten Konstitutionskonzepten zuarbeitete, die während der 1920er Jahre unter dem Einfluss holistischer Anschauungen auf die Konstitutionslehre an Bedeutung gewannen (Metzger 2017). Zu einem Konsens im Normproblem fand die Konstitutionslehre nie (vgl. Curtius 1954: 28-32).

In welchem Verhältnis standen nun die oben dargestellten anthropometrischen Reihenuntersuchungen zur Normdebatte? Die Untersuchungen von Brugsch $(1917,1918)$ und Berliner $(1920 a, b, 1921)$ waren von der Normdebatte noch unberührt. Beide wandten sich zwar gegen die Gleichsetzung von Norm und Durchschnittswerten, problematisierten aber nicht das Normkonzept als solches. Wenige Jahre später ging Brugsch (1922: 59), also während der bereits laufenden Normdebatte, dann kurz auf den konstruierten Charakter des durch Messreihen gewonnenen "Normotypus" ein, ohne sich auf eine nähere Problematisierung einzulassen. Vier Jahre später nahm er die Normdebatte an herausgehobener Stelle in sein Handbuch auf (Kaup 1926). An Rautmanns Beispiel ist besonders gut nachvollziehbar, dass seine Reihenmessungen - nach Plan seines Chefs de la Camp (1918: 516) - eigentlich dem Ziel dienen sollten, das „Normalindividuum“ mithilfe einer Konstitutionsformel näher zu beschreiben. Rautmann setzte im Vorwort seiner Studie auseinander, dass ihm während seiner Untersuchungen klar wurde, dass „die Unbestimmtheit des Begriffes ,normal ${ }^{\star}$ ganz besondere Schwierigkeiten verursacht" (Rautmann 1921: ix). Nicht nur müsse "normale Konstitution“ mit konkreten Inhalten gefüllt werden, auch sei der "Normbegriff einer eingehenden Kritik zu unterziehen“. So schrieb er nicht wie angedacht über „Proportionsbetrachtungen für verschiedene Typen“ (de la Camp 1918: 516), sondern Untersuchungen über die Norm. Ihre Bedeutung und Bestimmung. De la Camps Pläne für Rautmanns Reihenuntersuchungen wurden im Juni 1918 in der Berliner Klinischen Wochenschrift veröffentlicht - zu diesem Zeitpunkt vermaß Rautmann schon seit Monaten Soldaten in de la Camps Fliegeruntersuchungskommission (seit Frühherbst 1917; Rautmann 1921: 18). Er hatte also seine Reihenmessungen begonnen, ohne sich zunächst nähere Gedanken über das Wesen der zu untersuchenden „normalen Konstitution“ zu machen; frühestens im Verlauf der Messungen, vielleicht auch erst konfrontiert mit der Auswertung, drängte sich ihm die Normfrage auf, so dass er beschloss, dieser sein Buch zu widmen.

Rautmanns Monographie steht an einer Schalterstelle zwischen der Suche nach dem Normalkörper und der medizintheoretischen Problematisierung des Normbegriffs. Noch kurz vor Rautmann hatte der Würzburger Internist Richard Geigel (1859-1930) seine Tätigkeit in einer Fliegerun- 
tersuchungskommission genutzt, um einen „Kanon des jungen Soldaten“ aufzustellen, in dem ein lebhaftes Bild des gesunden und geistig-moralisch hochstehenden Durchschnittssoldaten gezeichnet wird (Geigel 1919). Um seine Durchschnittswerte zu erlangen, hatte er bei seinen Reihenmessungen 364 „vorzügliche“ Individuen ausgewählt - die subjektive Auswahl stützte er mit Verweis auf seine ärztliche Erfahrung und Autorität (Geigel 1919: 1491). Bereits wenige Jahre später verwiesen Konstitutionspathologen in der Normdebatte nur noch eher lächelnd auf Geigels Studie (Grote 1921: 8; Borchardt 1924: 6). Auch an Rautmanns Arbeit wurde kritisiert, dass seine Zahlen lediglich den ,jungen Flieger", nicht den "jungen Deutschen“ abbildeten (Grote 1922: 320; Rössle 1922: 209). Nachdem der Normbegriff erst einmal in der Konstitutionslehre problematisiert worden war, konnte niemand mehr hinter diese Entwicklung zurücktreten. Doch behinderte die Problematisierung des Normbegriffs auch klare Ergebnisse - es fiel schwer zu erforschen, was konzeptuell noch unklar blieb. Manche Autoren wie Bauer verabschiedeten sich vielleicht auch deshalb im Laufe der 1920er Jahre von der anthropometrischen Richtung der Konstitutionslehre, die in der Konstitutionsforschung fortan nur noch eine geringe Rolle spielen sollte.

\section{Von Sammellust bis Methodenreflexion - Fazit}

Vor dem Ersten Weltkrieg erhoffte sich die Konstitutionslehre von anthropometrischen Reihenmessungen sowohl eine methodische Verwissenschaftlichung ihrer Fächer, als auch die Beantwortung der zentralen Frage nach der „normalen“ Konstitution. Mit Beginn des Krieges ergriffen Konstitutionspathologen enthusiastisch die sich bietenden Gelegenheiten, entsprechende Reihenmessungen durchzuführen, ohne vorher ausführlich über Messmethoden, Vergleichs- oder Auswertungsmöglichkeiten zu reflektieren. Sie erhoben, wie am Beispiel der Brustumfangsmessung deutlich wurde, Messdaten an tausenden von Personen, die nicht mit den Messungen anderer Anthropometriker vergleichbar und damit beschränkt wissenschaftlich nutzbar waren. Aus dieser anfänglich unverhohlenen Sammellust entstand innerhalb sehr kurzer Zeit sowohl beim individuellen Forschenden als auch im Denkkollektiv der Konstitutionslehre eine Reflexion der Untersuchungs- und Auswertungsmethoden; Wert und Bedeutung der Statistik wurden erkannt, so dass innerhalb weniger Jahre die mathematischstatistischen Kenntnisse innerhalb der Konstitutionslehre stark anstiegen. Hatte man zunächst noch in der Variabilitätsstatistik eine Antwort darauf gesucht, wie der „Normotypus“ aussähe, wurde ab 1921 der Normbe- 
griff zunehmend problematisiert. Dies löste eine grundlegende Debatte zur „Norm" aus, die bis Mitte der 1920er Jahre unter Konstitutionspathologen intensiv geführt wurde.

Konstitutionspathologen sahen die Konstitutionslehre als methodischtheoretische Vorreiterin für die gesamte Medizin. Von Beginn an wollten sie naturwissenschaftlich ausgerichtete Forschungsmethoden in der Inneren Medizin anstoßen, wozu auch die anthropometrischen Untersuchungen des Untersuchungszeitraums gedacht waren. Dem Gebrauch mathematisch-statistischer Methoden stand man in der Konstitutionslehre von Anfang an aufgeschlossen gegenüber; ab 1921 führten dann die Werke von Rautmann, Bauer und Günther Novizen in diese Methode ein und bewerteten dabei Statistikanwendung als besonders wichtig für die Konstitutionsforschung. Der schnelle Zuwachs an statistischen Grundkenntnissen bei den untersuchten Internisten lässt darauf schließen, dass die Konstitutionslehre auf diesem Gebiet erfolgreich die Verbreitung statistischer Methoden unter ihren eigenen Vertretern angeregt hatte. Es ist also berechtigt, der Konstitutionslehre ihre Vorreiterrolle für die Verbreitung dieser Methoden auch objektiv zuzugestehen.

Ob dieser Kompetenzzuwachs auch langfristig wirkte, muss allerdings kritisch betrachtet werden. Innerhalb der Konstitutionslehre verloren anthropometrische Reihenuntersuchungen und deren statistische Auswertung im Laufe der 1920er Jahre schnell an Bedeutung. Dies mag zum einen in der starken Ausrichtung an holistischen Anschauungen begründet liegen, die große Teile der Konstitutionslehre zu prägen begannen (Timmermann 2001; Metzger 2017). Der holistische Blick auf die ganze Person des Patienten als Individuum verbot eine simple Charakterisierung durch einige Körpermaße oder gar einen Konstitutionsindex. Zum anderen verschoben sich die Forschungsfragen: Mit der aufkeimenden Normdebatte verlor die Frage nach einer in Zahlen fassbaren "normalen Konstitution“ an Bedeutung. So richtete die konstitutionspathologische Diskussion bis Ende der 1920er Jahre den Blick zunächst auf die Entstehung der individuellen Konstitution, dann auf mögliche Prophylaxe und Therapie (Metzger 2017). Die anthropometrische Variationsforschung ging schließlich fast ganz auf die Anthropologie über; doch auch in der Sportmedizin wurden, unter anderem durch Rautmann, ihre Methoden fortgeführt (Dinçkal 2013: 251-61; Bäumler 2010; Court 2002). Außerdem pflegten die Vererbungsforschung, selbst ein zentrales Anwendungsfeld mathematisch-statistischer Methoden (Desrosières 2005: 127-142; Schappacher \& Oehler-Klein 2007), und die im Nationalsozialismus geförderte Rassenhygiene personelle wie inhaltliche Kontinuitäten zur Konstitutionslehre, wie etwa Karl Heinrich Bauer (Metzger 2017: 298). 
Ob und inwiefern sich Statistikkenntnisse hingegen durch konstitutionspathologisch interessierte Internisten innerhalb der Inneren Medizin generell und langfristig verbreiten konnten, bleibt ein Forschungsdesiderat, darf aber beim jetzigen Forschungsstand bezweifelt werden: So stieß Anfang der 1930er Jahre die Methodenlehre der therapeutischen Untersuchung des Internisten Paul Martini (1889-1964) mit einer ihrer zentralen Forderungen, klinische Studien mithilfe von mathematisch-statistischen Methoden auszuwerten, zunächst auf wenig Resonanz (Martini 1932; Hofer 2019). Erst nach dem Zweiten Weltkrieg sollte Martinis Konzept, das heute gemeinhin als Vorläufer moderner klinischer Studien in Deutschland gilt, Gehör finden; die eigentliche Etablierung der randomisierten klinischen Studie als Methode in der BRD seit den 1960er Jahren erfolgte allerdings durch Rezeption englischsprachiger Forschung. Intensiv und öffentlichkeitswirksam wurde sie dann Ende der 1970er diskutiert, als die doppelverblindete klinische Studie Eingang ins Arzneimittelgesetz fand (Tröhler 1992: 72-74; Hofer 2019: 51). Bei Martini (1932) selbst finden sich keine Verweise auf die statistisch-methodischen Vorarbeiten aus der Konstitutionslehre. Dies deckt sich mit der These von Seydel (1976: 81), dass in Deutschland seit Beginn des 19. Jahrhunderts immer wieder ein Abreißen mathematischstatistischer Kenntnisse in der Medizin mit folgendem Neubeginn erfolgte. Letztlich setzten sich mathematisch-statistische Methoden in der BRD in der vollen Breite der medizinischen Disziplinen also erst in der zweiten Hälfte des 20. Jahrhunderts unter dem Druck der Internationalisierung sowie unter dem Einfluss neuer technischer Möglichkeiten durch. So ließ auch die Institutionalisierung der Medizinischen Statistik als Lehrfach noch Jahrzehnte lang auf sich warten. In der unmittelbaren Nachkriegszeit wurden statistische Methoden stark mit nationalsozialistischer Entmenschlichung assoziiert, was deren Etablierung in der Medizin stark behinderte (Hofer 2019: 49-50). Erst ab den 1960er Jahren wurden einzelne Professuren und Lehrstühle für Medizinische Statistik eingerichtet, so etwa 1963 in Mainz (vgl. 1972 Erlangen. Hofer 2019: 49; Schappacher \& Oehler-Klein 2007: 253).

Auch die konkreten gewonnenen Daten der anthropometrischen Untersuchungen durch Konstitutionspathologen hinterließen wenig Echo, da nicht nur unklare oder einheitliche Messmethoden sondern auch nicht repräsentative Stichproben - Kranke bei Berliner, Fliegerrekruten bei Rautmann - die Daten schlecht vergleichbar machten. Viele methodische Entscheidungen entsprachen nicht lange dem Forschungsstand, etwa Berliners Fokus auf Mittelwerte plus maximale Abweichungen oder Rautmanns Aufrunden von Messwerten auf ganze Zentimeter. Brugschs These von den drei Wuchshöhe-Typen konnte sich ebenfalls nicht durchsetzen; eigentlich alle folgenden Autoren tendierten dazu, eine Normalverteilung der 
Körpergröße anzunehmen. Insgesamt wirkte von den hier untersuchten anthropometrischen Studien Rautmann (1921) trotz gewisser Kritikpunkte am meisten nach, da sein Name langfristig mit dem Versuch verbunden wurde, einen statistischen Normbereich zu definieren (etwa Proppe 1954). Auch seine Daten für die Herzgröße wurden in der Nachkriegszeit noch rezipiert (Metzger 2020).

Obwohl die Konstitutionslehre ihre anthropometrische Suche nach dem „normalen“ Körper nur wenige Jahre lang betrieb, bevor sie sich davon abwandte, kommt ihr jedoch das Verdienst zu, die medizinische Normdebatte der 1920er Jahre angestoßen zu haben, die langfristig und über die Konstitutionslehre hinaus in die Medizintheorie Eingang finden sollte, insbesondere in Verbindung mit der verwandten Frage nach der Abgrenzung zwischen Krankheit und Gesundheit (Büttner 1997). Publikationen von Konstitutionspathologen zu diesen Fragen blieben wichtige Referenzpunkte für Mediziner, die sich in der Folgezeit zum Thema äußerten (etwa Proppe 1954; Müller-Suur 1950).

Insbesondere die fünf Jahre zwischen 1917 und 1922 stellten einen Drehpunkt in der Konstitutionslehre (und vielleicht auch darüber hinaus) dar, was den reflektierten Gebrauch von anthropometrischen und statistischen Methoden sowie des Normbegriffs angeht. Es konnte gezeigt werden, wie schnell sich die beteiligten Internisten hinsichtlich Anthropometrie, Statistik und Normproblem zunehmend professionalisierten, aber auch, auf welch basalem Niveau sie anfingen. Es zeichnet sich ein Bild ab von forschungswilligen Medizinern, die schnell zugriffen, als ihre militärärztliche Tätigkeit geeignetes Forschungsmaterial bot, ohne zunächst ihre Methoden genauer zu reflektieren oder sich im relevanten Forschungsstand außerhalb ihres eigenen Faches zu orientieren. Innerhalb von kurzer Zeit holten sie diese Defizite auf, aber gerade diese methodischen und medizintheoretischen Fortschritte machten viele der anthropometrischen Messreihen, wenige Jahre zuvor an tausenden von Personen erhoben, zu obsoleten Zahlensammlungen.

\section{Danksagung}

Mein Dank geht an die anonymen Gutachter*innen für ihre hilfreichen Hinweise und frischen Blick auf das Thema sowie an Susanne Ude-Koeller und Karl-Heinz Leven für ihre kritische Lektüre und fruchtbaren Diskussionen.

Funding Open Access funding provided by Projekt DEAL. 
Open Access Dieser Artikel wird unter der Creative Commons Namensnennung 4.0 International Lizenz veröffentlicht, welche die Nutzung, Vervielfältigung, Bearbeitung, Verbreitung und Wiedergabe in jeglichem Medium und Format erlaubt, sofern Sie den/die ursprünglichen Autor(en) und die Quelle ordnungsgemäß nennen, einen Link zur Creative Commons Lizenz beifügen und angeben, ob Änderungen vorgenommen wurden.

Die in diesem Artikel enthaltenen Bilder und sonstiges Drittmaterial unterliegen ebenfalls der genannten Creative Commons Lizenz, sofern sich aus der Abbildungslegende nichts anderes ergibt. Sofern das betreffende Material nicht unter der genannten Creative Commons Lizenz steht und die betreffende Handlung nicht nach gesetzlichen Vorschriften erlaubt ist, ist für die oben aufgeführten Weiterverwendungen des Materials die Einwilligung des jeweiligen Rechteinhabers einzuholen.

Weitere Details zur Lizenz entnehmen Sie bitte der Lizenzinformation auf http://creativecom mons.org/licenses/by/4.0/deed.de.

\section{Anmerkungen}

1 Dieser Aufsatz verwendet „Konstitutionslehre“ und „Konstitutionspathologie“ synonym; „Konstitutionpathologe“ und „konstitutionspathologisch“ werden davon abgeleitet.

2 Zur Unterscheidung zwischen deskriptiver Statistik („Staatsbeschreibung“) und mathematischer oder schließender Statistik (auf Basis der Wahrscheinlichkeitsrechnung) siehe Bilo et al. (2019: 21-22); historisch-zeitgenössisch Žižek (1923: 3-9).

3 Außerdem zur Konstitutionslehre siehe Probst (1982). In Bezug auf individualisierende Medizin Gadebusch Bondio \& Michl (2012: insbes. 122-131); Gadebusch Bondio (2015). In Bezug auf Vererbungslehre und Eugenik Baader (2007: insbes. 86-92); Hofer (2007); Weindling (1989: 170-75, 232-35); Hau (2003: 101-75). Aus der Perspektive der Bakteriologie Mendelsohn (2003).

4 Speziell mit der Normdebatte der 1920er Jahre beschäftigen sich Büttner $(1997,1998)$; Felsch (2005); Gadebusch Bondio (2015); Stukenbrock (2002); Vácha (1985). Zum Zusammenhang zwischen metrischen Verfahren und Normierung siehe die Aufsätze in den Sammelbänden von Hess (1997) und Borck et al. (2005); siehe außerdem Hess (1999, 2000); sowie Fangerau \& Martin (2014) als Beispiele aus der reichen Forschungsliteratur.

5 Zur Anwendung von mathematisch-statistischen Methoden in der klinischen Medizin speziell in den deutschsprachigen Ländern während des Untersuchungszeitraums wurde nur wenig publiziert. Herauszuheben sind Büttner (1997); Nikolow (1997); Tröhler (1992); Seydel (1976). Weiterhin Martin \& Fangerau (2010). International etwa Jorland et al. (2005). Gut erforscht ist hingegen die Medizinalstatistik für Kaiserreich und Weimarer Republik, die allerdings die mathematisch-statistischen Methoden kaum anwandte, mit denen sich die vorliegende Arbeit beschäftigt, und weitgehend deskriptiv arbeitete. Dazu etwa Hüntelmann (2008) und Schneider (2006); in Bezug auf die militärische Musterung Hartmann (2011). Einen aktuellen Forschungsüberblick bietet Hüntelmann (2019: 26-27). Einführend in das aktuelle Forschungsfeld Bilo et al. (2019).

6 Zur Anthropometrie siehe etwa Morris-Reich (2013) zu Rudolf Martin; zur Vermessung bei der militärischen Musterung Hartmann (2011); zur Anthropometrie der frühen Sportwissenschaft Dinçkal (2013: 251-61) und Court (2002); zur anthropometrischen Konstitutionsforschung in Italien Albrizio (2007). Ein Klassiker zur Anthropometrie ist Gould (1999).

7 Speziell zu den hier untersuchten Reihenmessungen durch konstitutionsinteressierte Internisten siehe Stukenbrock (2002: 55-60).

8 Kritiker einer statistischen Lösung des Konstitutionsproblems wie der Pädiater Pfaundler (1911: 46) blieben innerhalb der Konstitutionslehre in den Anfangsjahren in der Min- 
derheit, obwohl Pfaundler Statistik für andere Fragestellungen sehr wohl einsetzte (etwa Pfaundler 1916).

9 Brugsch schweigt sich in seinen ersten Veröffentlichungen der Reihenmessungen zur genauen Herkunft der Daten aus. Erst in einer späteren Monographie spezifiziert er in einer Fußnote die genaue Entstehungszeit und den militärischen Kontext (Brugsch 1922: 82).

10 Zu Friedrich Martius (1850-1923) und Friedrich Kraus (1858-1936) als frühe Konstitutionspathologen siehe Krügel (1984); Lindner (1999); Metzger (2016). Bauer wirkte als ein wichtiger Sachwalter der Konstitutionslehre (Metzger 2017); zu seiner Person siehe Forsbach \& Hofer (2018: 90-93); Hofer (2007). Der Leipziger Internist Hans Günther (1884-1956), 1921 habilitiert, hatte ab 1928 ebenda die erste dezidierte Professur für Konstitutionsforschung inne. Er ist nicht identisch mit dem späteren „Rasse-Günther“, Hans F. K. Günther (Fischer 1962; Voswinckel 2002).

11 Ablehnend bis distanziert etwa die von Rautmann rezipierten Arbeiten von Bruns sowie Ranke \& Greiner. Bruns erwähnte in seinem Lehrbuch Pearson und dessen Korrelationskoeffizienten nur zwei Mal und das ablehnend (1906: 160n1 u. 111), er orientierte sich wie Rautmann stark an Fechner. Ranke \& Greiner (1904) besprachen Pearson zwar ausführlich, distanzierten sich aber explizit, da Pearsons Beiträge für die Anthropologie nutzlos seien - so seien etwa dessen „Ableitungen [...] als mit dem Geschehen in der Natur unvereinbar" aufzufassen (Ranke \& Greiner 1904: 324). Rautmann sah das zwar nicht so (1921: 103n2), aber benutzte auch keine Pearson'schen Konzepte.

\section{Literatur}

Albrizio, Angelo 2007. Biometry and Anthropometry. From Galton to Constitutional Medicine. Journal of Anthropological Sciences (85): 101-123.

Aschoff, Ludwig 1916. Über die Aufgaben der Kriegspathologie. In: M.B. Schmidt und W. Berblinger (Hg.). Kriegspathologische Tagung in Berlin am 26. und 27. April 1916. Beiheft zu Centralblatt für allgemeine Pathologie und pathologische Anatomie. Bd. 27. Jena: Gustav Fischer: 1-9.

Baader, Gerhard 2007: Eugenische Programme in der sozialistischen Parteienlandschaft in Deutschland und Österreich im Vergleich. In: Gerhard Baader, Veronika Hofer und Thomas Mayer (Hg.). Eugenik in Österreich. Biopolitische Strukturen von 1900-1945. Wien: Czernin: 66-139.

Bauer, Julius 1918. Beiträge zur klinischen Konstitutionspathologie I. Habitus und Morbidität I. Deutsches Archiv für klinische Medizin (126): 196-223.

Bauer, Julius 1921. Vorlesungen über allgemeine Konstitutions- und Vererbungslehre für Studierende und Ärzte. Berlin: Julius Springer.

Bauer, Julius 1923. Vorlesungen über allgemeine Konstitutions- und Vererbungslehre für Studierende und Ärzte. 2. Aufl. Berlin: Julius Springer.

Bäumler, Günther 2010. Die Rezeption des Korrelationskoeffizienten in der deutschen sportwissenschaftlichen Forschung des frühen 20. Jahrhunderts. Eine Dokumentation. In: Jürgen Court, Arno Müller und Andrea Schulte (Hg.). Jahrbuch 2008 der Deutschen Gesellschaft für Geschichte der Sportwissenschaft e. V. Berlin: LIT: 66-129.

Berliner, Max 1920a. Ueber die Beziehungen des proportionellen Brustumfangs zum Index der Körperfülle bei männlichen Individuen im Wachstumsalter. Berliner klinische Wochenschrift (57): 33-34.

Berliner, Max 1920b. Die Bedeutung der Anthropometrie für die Klinik. Zeitschrift für experimentelle Pathologie und Therapie (21): 341-357.

Berliner, Max 1921. Untersuchungen über die Beziehungen zwischen Thoraxform und Gesamtorganisation. Zeitschrift für experimentelle Pathologie und Therapie (22): 135-151. 
Berliner, Max, Theodor Brugsch und Alfred Schittenhelm 1923. Körpermessung. In: Theodor Brugsch und Alfred Schittenhelm (Hg.). Klinische Laboratoriumstechnik. Bd. 1. Berlin: Urban \& Schwarzenberg: 1-27.

Bilo, Nicolas, Stefan Haas und Michael C. Schneider 2019. Grundbegriffe und Konturen einer Kulturgeschichte der Statistik. Einleitung. In: Dieselben (Hg.). Die Zählung der Welt. Kulturgeschichte der Statistik vom 18. bis 20. Jahrhundert. Stuttgart: Steiner: 9-22.

Borchardt, Leo 1924. Klinische Konstitutionslehre. Ein Lehrbuch für Studierende und Ärzte. Berlin: Urban \& Schwarzenberg.

Borchardt, Leo 1930. Klinische Konstitutionslehre. Ein Lehrbuch über die Beziehungen zwischen Körperverfassung und Krankheit für Studierende und Ärzte. 2. Aufl. Berlin: Urban \& Schwarzenberg.

Borck, Cornelius, Volker Hess und Henning Schmidgen (Hg.) 2005. Maß und Eigensinn. Studien in Anschluß an Georges Canguilhem. München: Wilhelm Fink.

Brugsch, Theodor 1917. Masse und Proportionen zur Charakterisierung des Individuums in seinem Habitus. Zeitschrift für experimentelle Pathologie und Therapie (19): 1-15.

Brugsch, Theodor 1918. Allgemeine Prognostik oder die Lehre von der ärztlichen Beurteilung des gesunden und kranken Menschen. Berlin: Urban \& Schwarzenberg.

Brugsch, Theodor 1922. Allgemeine Prognostik oder die Lehre von der ärztlichen Beurteilung des gesunden und kranken Menschen. 2. Aufl. Berlin: Urban \& Schwarzenberg.

Brugsch, Theodor und Alfred Schittenhelm 1918. Lehrbuch klinischer Untersuchungsmethoden für Studierende und Ärzte. 4. Aufl. Berlin: Urban \& Schwarzenberg.

Bruns, Heinrich 1906. Wahrscheinlichkeitsrechnung und Kollektivmasslehre. Berlin: Teubner.

Büttner, Johannes 1997. Die Herausbildung des Normalwert-Konzeptes im Zusammenhang mit quantitativen diagnostischen Untersuchungen in der Medizin. In: Volker Hess (Hg.). Die Normierung der Gesundheit. Messende Verfahren der Medizin als kulturelle Praxis um 1900. Husum: Matthiesen: 17-32.

Büttner, Johannes 1998. Biological Variation and Quantification of Health. The Emergence of the Concept of Normality. Clinical Chemistry and Laboratory Medicine (36): 69-73.

De la Camp, Oskar 1918. Beitrag zu konstitutionellen Mittelwerten. Berliner Klinische Wochenschrift (55): 515-517.

Court, Jürgen 2002. Sportanthropometrie und Sportpsychologie in der Weimarer Republik. Sportwissenschaft (32): 401-414.

Curtius, Friedrich 1954. Klinische Konstitutionslehre. Berlin: Springer.

Czuber, Emanuel 1908. Wahrscheinlichkeitsrechnung und ihre Anwendung auf Fehlerausgleichung, Statistik und Lebensversicherung. Bd. 1: Wahrscheinlichkeitstheorie, Fehlerausgleichung, Kollektivmasslehre. 2. Aufl. Leipzig: Teubner.

Desrosières, Alain 2005. Die Politik der großen Zahlen. Eine Geschichte der statistischen Denkweise. Berlin: Springer.

Dikanski, Mordchaj 1914. Ueber den Einfluss der sozialen Lage auf die Körpermaasse von Schulkindern. Med. Dissertation, München: Ludwig-Maximilians-Universität.

Dinçkal, Noyan 2013. Sportlandschaften. Sport, Raum und (Massen-)Kultur in Deutschland 1880-1930. Göttingen: Vandenhoeck \& Ruprecht.

Eckart, Wolfgang U. 2014. Medizin und Krieg. Deutschland 1914-1924. Paderborn: Schöningh.

Eckart, Wolfgang U. und Christoph Gradmann 1996 (Hg.). Die Medizin und der Erste Weltkrieg. Pfaffenweiler: Centaurus.

Fangerau, Heiner und Michael Martin 2014. Blutdruck messen. Die „Technikalisierung“ der Kreislaufdiagnostik. In: Alexander Sigelen und Wolf-Diether Burak (Hg.). Herzblut. Geschichte und Zukunft der Medizintechnik. Katalog zur Großen Landesausstellung 2014 Baden-Württemberg. Darmstadt: Theiss: 74-92.

Fechner, Gustav Theodor 1897. Kollektivmasslehre. Hg. v. Gottlob Friedrich Lipps, Leipzig: Wilhelm Engelmann.

Felsch, Philipp 2005. Ganzheit und ihre Apparaturen. Konstitutionelle Medizin um 1900. In: Cornelius Borck, Volker Hess und Henning Schmidgen (Hg.). Maß und Eigensinn. Studien im Anschluß an Georges Canguilhem. München: Wilhelm Fink: 135-156. 
Fischer, Isidor 1962. Art. Günther, Hans. In: Derselbe. Biographisches Lexikon der hervorragenden Ärzte der letzten fünfzig Jahre. Nachdruck: Bd. 1. München, Berlin: Urban \& Schwarzenberg 1932-1933: 549.

Florschütz, Georg 1914. Allgemeine Lebensversicherungsmedizin. Berlin: Mittler und Sohn.

Forsbach, Ralf und Hans-Georg Hofer 2018. Internisten in Diktatur und junger Demokratie. Die Deutsche Gesellschaft für Innere Medizin 1933-1970. Berlin: Medizinisch Wissenschaftliche Verlagsgesellschaft.

Frölich, F. Hermann 1872. Das zweckmässigste Brustmessungsverfahren. Archiv für pathologische Anatomie und Physiologie und für klinische Medicin (54): 352-375.

Gadebusch Bondio, Mariacarla 2015. „Das Individuum - eine Abweichung“ ... und das Unbehagen der Wissenschaft. In: Mariacarla Gadebusch Bondio und Eva Brinkschulte (Hg.). Norm als Zwang, Pflicht und Traum. Normierende versus individualisierende Bestrebungen in der Medizin. Frankfurt/Main: Peter Lang: 19-50.

Gadebusch Bondio, Mariacarla und Susanne Michl 2012. Von der Medikalisierung des Humanen. Das Individuelle als Herausforderung. In: Mariacarla Gadebusch Bondio und Hania Siebenpfeiffer (Hg.). Konzepte des Humanen. Ethische und kulturelle Herausforderungen. Freiburg/Breisgau: Alber: 117-38.

Geigel, Richard 1919. Der Kanon des jungen Soldaten. Münchener Medizinische Wochenschrift (66): 1491-1493.

Gould, Benjamin Apthorp 1869. Investigation in the Military Anthropological Statistics of American Soldiers. New York: Hurd \& Houghton.

Gould, Stephen Jay 1999. Der falsch vermessene Mensch. 3. Aufl. Frankfurt/Main: Suhrkamp.

Groedel, Franz 1919. Der Durchschnittswert des Blutdrucks beim gesunden Menschen. Berliner Klinische Wochenschrift (56): 52-54.

Grote, Louis R. 1921. Grundlagen ärztlicher Betrachtung. Einführung in begriffliche und konstitutionspathologische Fragen der Klinik für Studierende und Ärzte. Berlin: Springer.

Grote, Louis R. 1922. Rez. Hermann Rautmann, Untersuchungen über die Norm. Zentralblatt für Innere Medizin (43): 319-320.

Gruber, Max von und Ernst Rüdin 1911. Fortpflanzung. Vererbung. Rassenhygiene. Illustrierter Führer durch die Gruppe Rassenhygiene der Internationalen Hygiene-Ausstellung 1911 in Dresden. 2. Aufl. München: Lehmanns.

Günther, Hans 1922. Die Grundlagen der biologischen Konstitutionslehre. Leipzig: Georg Thieme.

Harrington, Anne (Hg.) 1999. Reenchanted Science. Holism in German Culture from Wilhelm II to Hitler. Princeton: Princeton University Press.

Hartmann, Heinrich 2011. Der Volkskörper bei der Musterung. Militärstatistik und Demographie in Europa vor dem Ersten Weltkrieg. Göttingen: Wallstein.

Hau, Michael 2000. The Holistic Gaze in German Medicine, 1890-1930. Bulletin of the History of Medicine (74): 495-524.

Hau, Michael 2003. The Cult of Health and Beauty in Germany. A Social History 1890-1930. Chicago: University of Chicago Press.

Hess, Volker (Hg.) 1997. Normierung der Gesundheit. Messende Verfahren der Medizin als kulturelle Praxis um 1900. Husum: Matthiesen.

Hess, Volker 1999. Messen und Zählen. Die Herstellung des normalen Menschen als Maß der Gesundheit. Berichte zur Wissenschaftsgeschichte (22): 266-280.

Hess, Volker 2000. Der wohltemperierte Mensch. Fiebermessen in Wissenschaft und Alltag 1850-1900. Frankfurt/Main: Campus.

His, Wilhelm 1911. Geschichtliches und Diathesen in der Inneren Medizin. Verhandlungen des Deutschen Kongresses für Innere Medizin (28): 15-35.

Hofer, Hans-Georg 2002. Die „Veränderung aller Maßstäbe“. Die Freiburger Medizinische Fakultät und der Erste Weltkrieg. In: Bernd Grün, Hans-Georg Hofer und Karl-Heinz Leven (Hg.). Medizin und Nationalsozialismus. Die Freiburger Medizinische Fakultät und das Klinikum in der Weimarer Republik und im „Dritten Reich“. Frankfurt/Main: Peter Lang: 50-75.

Hofer, Hans-Georg 2019. Der Arzt als therapeutischer Forscher. Paul Martini und die Verwissenschaftlichung der klinischen Medizin. Acta Historica Leopoldina (74): 41-59.

Hofer, Veronika 2007. Positionen und Posen eines Experten. Der Konstitutionsforscher Julius Bauer (1887-1973) und die Eugenik in der Wiener Zwischenkriegszeit. In: Gerhard 
Baader, Veronika Hofer und Thomas Mayer (Hg.). Eugenik in Österreich. Biopolitische Strukturen von 1900-1945. Wien: Czernin: 31-65.

Hoßfeld, Uwe 2005: Geschichte der biologischen Anthropologie in Deutschland. Von den Anfängen bis in die Nachkriegszeit. Stuttgart: Steiner.

Hüntelmann, Axel C. 2008. Hygiene im Namen des Staates. Das Reichsgesundheitsamt 1876-1933. Göttingen: Wallstein.

Hüntelmann, Axel C. 2019. Konstruktion und Etablierung der Medizinalstatistik in Deutschland. Ca. 1850-1900. In: Stefan Haas, Michael C. Schneider und Nicolas Bilo (Hg.). Die Zählung der Welt. Kulturgeschichte der Statistik vom 18. bis 20. Jahrhundert. Stuttgart: Steiner: 23-49.

Johannsen, Wilhelm 1909. Elemente der exakten Erblichkeitslehre. Deutsche wesentlich erweiterte Ausgabe in fünfundzwanzig Vorlesungen. Jena: Gustav Fischer.

Jorland, Gérard, Annick Opinel und George Weisz (Hg.) 2005. Body counts. Medical quantification in historical and sociological perspective. Montréal: McGill-Queen's University Press.

Kaup, Ignaz 1922. Untersuchungen über die Norm. Münchener Medizinische Wochenschrift (69): 189-192.

Kaup, Ignaz 1926. Bedeutung des Normbegriffs in der Personallehre. In: Theodor Brugsch und Friedrich H. Lewy (Hg.). Die Biologie der Person. Ein Handbuch der allgemeinen und speziellen Konstitutionslehre. Bd. 1. Berlin: Urban \& Schwarzenberg: 191-225.

Kisskalt, Karl 1919. Einführung in die Medizinalstatistik in praktischen Übungen zur Benutzung in Kursen und zum Selbstunterricht. Leipzig: Georg Thieme.

Kraus, Friedrich 1917. Körpermaß und Körperproportion im Zusammenhang mit Entwicklung, Wachstum und Funktion als Gegenstand der Konstitutionslehre. Mit spezieller Berücksichtigung der militärärztlichen Begutachtung. In: Curt Adam (Hg.). Die militärärztliche Sachverständigentätigkeit auf dem Gebiete des Ersatzwesens und der militärischen Versorgung. Hrsg. v. Zentralkomitee für das ärztliche Fortbildungswesen in Preußen. 2. Teil. Jena: Gustav Fischer: 241-296.

Krehl, Ludolf 1911. Eröffnungsrede. Verhandlungen des Deutschen Kongresses für Innere Medizin (28): 3-11.

Krügel, Rainer 1984. Friedrich Martius und der konstitutionelle Gedanke. Frankfurt/Main: Peter Lang.

Lang, Arnold 1914: Die experimentelle Vererbungslehre in der Zoologie seit 1900. Ein Sammelwerk und Hilfsbuch bei Untersuchungen. Bd. 1. Jena: Gustav Fischer.

Lawrence, Christopher und George Weisz (Hg.) 1998. Greater Than the Parts. Holism in Biomedicine, 1920-1950. New York: Oxford University Press.

Lindner, Martin 1999. Die Pathologie der Person. Friedrich Kraus' Neubestimmung des Organismus am Beginn des 20. Jahrhunderts. Berlin: Verlag für Geschichte der Naturwissenschaften und der Technik.

Lubarsch, Otto 1921. Zur Konstitutions- und Dispositionslehre. Die Naturwissenschaften (9): 812-819.

Martin, Rudolf 1914. Lehrbuch der Anthropologie in systematischer Darstellung. Mit besonderer Berücksichtigung der anthropologischen Methoden. Für Studierende, Ärzte und Forschungsreisende. Jena: Gustav Fischer.

Martin, Rudolf 1922. Anthropometrie. Münchener Medizinische Wochenschrift (69): 383389.

Martin, Rudolf 1924. Richtlinien für Körpermessungen und deren statistische Verarbeitung mit besonderer Berücksichtigung von Schülermessungen. München: Lehmanns.

Martin, Michael und Heiner Fangerau 2010. Claude Bernard und der „europäische Durchschnittsharn". Der Urologe (49): 855-860.

Martini, Paul 1932. Methodenlehre der therapeutischen Untersuchung. Berlin: Julius Springer.

Martius, Friedrich 1909. Pathogenese innerer Krankheiten. Nach Vorlesungen für Studierende und Ärzte. 2. Aufl. Leipzig, Wien: Deuticke.

Martius, Friedrich 1914. Konstitution und Vererbung in ihren Beziehungen zur Pathologie. Berlin: Julius Springer.

Massin, Benoît 2003. Rasse und Vererbung als Beruf. Die Hauptforschungsrichtungen am Kaiser-Wilhelm-Institut für Anthropologie, menschliche Erblehre und Eugenik 
im Nationalsozialismus. In: Hans-Walter Schmuhl (Hg.). Rassenforschung an KaiserWilhelm-Instituten vor und nach 1933. Göttingen: Wallstein: 190-244.

Mendelsohn, J. Andrew 2003. Medicine and the making of bodily inequality in twentiethcentury Europe. In: Jean-Paul Gaudillière und Ilana Löwy (Hg.). Heredity and Infection. The History of Disease Transmission. London: Routledge: 21-79.

Metzger, Nadine 2016. „Auf strengster wissenschaftlicher Grundlage“. Die Etablierungsphase der modernen Konstitutionslehre 1911 bis 1921. Medizinhistorisches Journal (51): 209-245.

Metzger, Nadine 2017. „Es sind noch große Forschungserträge zu erhoffen.“ Entwicklungen der Konstitutionslehre in den 1920er Jahren. Medizinhistorisches Journal (52): 270-307.

Metzger, Nadine 2020. Der Internist, Konstitutionspathologe und Sportmediziner Hermann Rautmann (1885-1956). Sudhoffs Archiv (104: im Druck).

Morris-Reich, Amos 2013. Anthropology, standardization and measurement. Rudolf Martin and anthropometric photography. British Journal for the History of Science (46): $487-516$.

Müller-Suur, Hemmo 1950. Das Psychisch Abnorme. Untersuchungen zur allgemeinen Psychiatrie. Berlin: Springer.

Neuburger, Max 1914. Zur Geschichte der Konstitutionslehre. Zeitschrift für angewandte Anatomie und Konstitutionslehre (1): 4-10.

Nikolow, Sybilla 1997. Die Mathematizität von Blut und Urin. Statistische Methoden in der Medizin. Kommentar. In: Volker Hess (Hg.). Normierung der Gesundheit. Messende Verfahren der Medizin als kulturelle Praxis um 1900. Husum: Matthiesen: 33-37.

Nikolow, Sybilla 2001. Der statistische Blick auf Krankheit und Gesundheit. „Kurvenlandschaften" in Gesundheitsausstellungen am Beginn des 20. Jahrhunderts in Deutschland. In: Ute Gerhard, Jürgen Link und Ernst Schulte-Holtey (Hg.). Infografiken, Medien, Normalisierung. Zur Kartografie politisch-sozialer Landschaften. Heidelberg: Synchron: 223-241.

Oeder, Gustav 1915a. 281 erwachsene Menschen mit "centralnormalem“ Ernährungszustand. Berliner Klinische Wochenschrift (52): 433-438, 466-470.

Oeder, Gustav 1915b. Die Gärtner'sche Normalgewichtstabelle für Erwachsene. Berliner Klinische Wochenschrift (52): 1086-1092.

Pagel, Julius 1901. Art. Frölich, F. Hermann. In: Julius Pagel. Biographisches Lexikon hervorragender Ärzte des neunzehnten Jahrhunderts. Mit einer historischen Einleitung. Berlin: Urban \& Schwarzenberg: 560-562.

Pawliczek, Aleksandra 2011. Akademischer Alltag zwischen Ausgrenzung und Erfolg. Jüdische Dozenten an der Berliner Universität 1871-1933. Stuttgart: Steiner.

Pfaundler, Meinhard von 1911. Diathesen in der Kinderheilkunde. Verhandlungen des Deutschen Kongresses für Innere Medizin (28): 36-85.

Pfaundler, Meinhard von 1916. Körpermaß-Studien an Kindern. Zeitschrift für Kinderheilkunde (14): 1-148.

Porter, Theodore M. 1995. Trust in Numbers. The Pursuit of Objectivity in Science and Publicity Life. Princeton: Princeton University Press.

Probst, Johannes 1982. Zur Entwicklung der Konstitutionslehre zwischen 1911 und 1980. Med. Dissertation, Freiburg/Breisgau: Albert-Ludwigs-Universität.

Proppe, Albin 1954. Der Normbegriff in der Medizin. Ärztliche Wochenschrift (9): 217-221.

Prüll, Cay-Rüdiger [Livia] 1996. Die Sektion als letzter Dienst am Vaterland. Die deutsche „Kriegspathologie“ im Ersten Weltkrieg. In: Wolfgang U. Eckart und Christoph Gradmann (Hg.). Die Medizin und der Erste Weltkrieg. Pfaffenweiler: Centaurus: 155-182.

Prüll, Cay-Rüdiger [Livia] 2003. Medizin am Toten oder am Lebenden? Pathologie in Berlin und in London, 1900-1945. Basel: Schwabe.

Quetelet, Adolphe 1838. Ueber den Menschen und die Entwicklung seiner Fähigkeiten oder Versuch einer Physik der Gesellschaft. Stuttgart: Schweizerbart.

Ranke, Johannes 1911-1912. Der Mensch. 2 Bde. 3. Aufl. Leipzig: Bibliographisches Institut.

Ranke, Karl Ernst und Dr. Greiner 1904. Das Fehlergesetz und seine Verallgemeinerung durch Fechner und Pearson in ihrer Tragweite für die Anthropologie. Archiv für Anthropologie N.F. (2): 295-332. 
Rautmann, Hermann 1921. Untersuchungen über die Norm. Ihre Bedeutung und Bestimmung. Jena: Gustav Fischer.

Rautmann, Hermann 1923. Klinische Medizin und Variationsforschung. Münchener Medizinische Wochenschrift (70): 1355-1358.

Rautmann, Hermann 1924. Zur ärztlichen Untersuchung der deutschen Studentenschaft. Deutsche medizinische Wochenschrift (50): 565-69.

Riedel, Eduard 1913. Die Körperlänge von Münchner Schulkindern. Dargelegt nach den Prinzipien der Kollektivmaßlehre. Med. Dissertation, München: Ludwig-Maximilians-Universität.

Rössle, Robert 1919. Allgemeine Pathologie und Pathologische Anatomie. Bedeutung und Ergebnisse der Kriegspathologie. Jahreskurse für ärztliche Fortbildung (10): 15-46.

Rössle, Robert 1922. Rez. Hermann Rautmann, Untersuchungen über die Norm. Münchener Medizinische Wochenschrift (59): 208-209.

Ruppel, Irmgard 2009. Erinnerungen. München: FinanzBuch.

Salinger, Hans 1926. Die rechnerische Auswertung statistischer Beobachtungsergebnisse (Einführung in die Kollektivmaßlehre). In: Theodor Brugsch und Friedrich H. Lewy (Hg.). Die Biologie der Person. Ein Handbuch der allgemeinen und speziellen Konstitutionslehre. Bd. 1. Berlin: Urban \& Schwarzenberg: 135-190.

Schagen, Udo 2008. Wer wurde vertrieben? Wie wenig wissen wir? Die Vertreibungen aus der Berliner Medizinischen Fakultät 1933. Ein Überblick. In: Sabine Schleiermacher und Udo Schagen (Hg.). Die Charité im Dritten Reich. Zur Dienstbarkeit medizinischer Wissenschaft im Nationalsozialismus. Paderborn: Schöningh: 51-66.

Schappacher, Norbert und Sigrid Oehler-Klein 2007. Siegfried Koller und die neuen Herausforderungen der Statistik im Nationalsozialismus. In: Sigrid Oehler-Klein (Hg.). Die Medizinische Fakultät der Universität Gießen im Nationalsozialismus und in der Nachkriegszeit. Personen und Institutionen, Umbrüche und Kontinuitäten. Stuttgart: Steiner: 247-262.

Scheidt, Walter 1921. Zur Technik der Brustumfangmessung. Die Kindertuberkulose (1): 57-59.

Schmuhl, Hans-Walter 2005. Grenzüberschreitungen. Das Kaiser-Wilhelm-Institut für Anthropologie, menschliche Erblehre und Eugenik 1927-1945. Göttingen: Wallstein.

Schneider, Michael C. 2006. Medizinalstatistik im Spannungsfeld divergierender Interessen. Kooperationsformen zwischen statistischen Ämtern und dem Kaiserlichen Gesundheitsamt/Reichsgesundheitsamt. In: Axel C. Hüntelmann, Johannes Vossen und Herwig Czech (Hg.). Gesundheit und Staat. Studien zur Geschichte der Gesundheitsämter in Deutschland, 1870-1950. Husum: Matthiesen: 49-62.

Seydel, Hiltrud 1976. Statistik in der Medizin. Ein Entwurf zu ihrer Geschichte. Neumünster: Karl Wachholz.

Skibinski, Awrum 1914. Das Körpergewicht von Münchner Schulkindern. Med. Dissertation, München: Ludwig-Maximilians-Universität.

Stieda, Ludwig 1883. Ueber die Anwendung der Wahrscheinlichkeitsrechnung in der anthropologischen Statistik. Archiv für Anthropologie (14): 167-182.

Stukenbrock, Karin 2002. Individuum oder Gruppe. Mediziner auf der Suche nach dem normierten Menschen. In: Ulf-Christian Ewert, Stephan Selzer (Hg.). Menschenbilder Menschenbildner. Individuum und Gruppe im Blick des Historikers. Berlin: Akademie: 53-66.

Tandler, Julius 1914. Konstitution und Rassenhygiene (Vortrag Deutsche Gesellschaft für Rassenhygiene, 7. März 1914). Zeitschrift für angewandte Anatomie und Konstitutionslehre (1): 11-26.

Timmermann, Carsten 1996. Concepts of the Human Constitution in Weimar Medicine, 1918-1933. MA thesis, University Manchester.

Timmermann, Carsten 2001. Constitutional Medicine, Neoromanticism, and the Politics of Antimechanism in Interwar Germany. Bulletin of the History of Medicine (75): 717-739.

Tröhler, Ulrich 1992. Die therapeutische „Erfahrung“. Geschichte ihrer Bewertung zwischen subjektiv sicherem Wissen und objektiv wahrscheinlichen Kenntnissen. In: Johannes Köbberling (Hg.). Die Wissenschaft in der Medizin. Selbstverständnis und Stellenwert in der Gesellschaft. Stuttgart: Schattauer: 65-81. 
Vierordt, Hermann 1906. Anatomische physiologische und physikalische Daten und Tabellen. Zum Gebrauche für Mediziner. 3. Aufl. Jena: Gustav Fischer.

Voswinckel, Peter 2002. Günther, Hans. In: Isidor Fischer. Biographisches Lexikon der hervorragenden Ärzte der letzten fünfzig Jahre bearbeitet und herausgegeben von Peter Voswinckel. Bd. 3: Nachträge und Ergänzungen. Hildesheim: Georg Olms: 557.

Vácha, Jiří 1985. German Constitutional Doctrine in the 1920s and 1930s and Pitfalls of the Contemporary Conception of Normality in Biology and Medicine. Journal of Medicine and Philosophy (10): 339-367.

Weindling, Paul 1989. Health, Race and German Politics Between National Unification and Nazism 1870-1945. Cambridge: Cambridge University Press.

Zabel, Hans-Henning 1999. Orenstein, Benno. Neue Deutsche Biographie (19): 587-588.

Žižek, Franz 1923. Grundriss der Statistik. 2. Aufl. München: Duncker \& Humblot.

Hinweis des Verlags Der Verlag bleibt in Hinblick auf geografische Zuordnungen und Gebietsbezeichnungen in veröffentlichten Karten und Institutsadressen neutral.

\section{Nadine Metzger}

Lehrstuhl für Geschichte der Medizin

Friedrich-Alexander-Universität Erlangen-Nürnberg

Glückstr. 10

91054 Erlangen

Deutschland

nadine.metzger@fau.de 